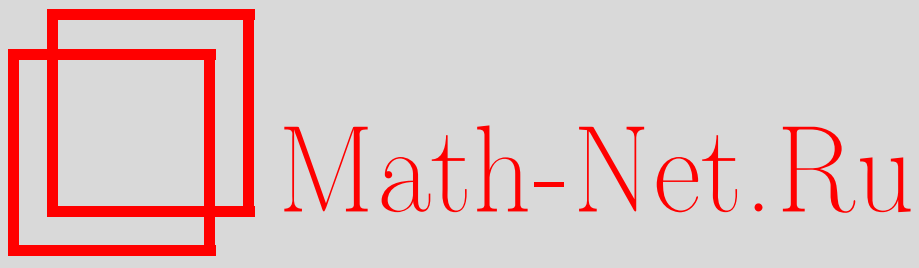

А. Р. Данилин, Асимптотика решений системы сингулярных эллиптических уравнений в прямоугольнике, Матем. сб., 2003, том 194, номер 1, 31-60

DOI: https://doi.org/10.4213/sm705

Использование Общероссийского математического портала Math-Net.Ru подразумевает, что вы прочитали и согласны с пользовательским соглашением

http://www . mathnet.ru/rus/agreement

Параметры загрузки:

IP: 3.80 .253 .173

26 апреля 2023 г., 15:01:56 
УДК 517.977

\author{
А.Р. Данилин
}

\title{
Асимптотика решений системы сингулярных эллиптических уравнений в прямоугольнике
}

\begin{abstract}
Рассматривается система из двух сопряженных эллиптических уравнений с малым параметром при старших производных в прямоугольнике, две стороны которого параллельны характеристикам предельных уравнений.

Методом согласования асимптотических разложений строятся равномерные асимптотики решения этой системы до любой степени малого параметра

Библиограф̆ия: 18 названий.
\end{abstract}

\section{Введение}

В работе методом согласования асимптотических разложений [1] строится равномерная асимптотика решений задачи Дирихле одной системы сингулярно возмущенных эллиптических уравнений со скалярньм параметром и дополнительным интегральным ограничением. K такой задаче сводится нахождение оптимального управления и соответствуюшего ему состояния в задаче оптимального управления решениями краевой задачи для эллиптического уравнения с малым коэффициентом при старших производных в области с частью границы, параллельной характеристике предельного уравнения [2]. Результаты работы анонсированы в [3], а предельные соотношения для этой задачи рассмотрены в [4].

Другой вид этой задачи рассмотрен в [5], [6]. Сингулярные задачи для уравнения эллиптического типа с малым коэффициентом при старшей производной в области с частью гранищы, параллельной характеристике, рассматривались также в $[7]-[9]$.

Построению асимптотических разложений различных сингулярно возмущенных задач, связанных с уравнениями в частных производных, посвящено значительное число исследований (см. обзор [10]). В теории оптимального управления, где сингулярно возмущенные задачи тоже вызьвают значительный интерес (см. [11]-[14]), условия оптимальности часто приводят к необходимости нахождения решений систем из двух сопряженных (в некотором смысле) уравнений [2]. Это вносит существенные трудности в осуществление известных подходов к построению асимптотических разложений решений. Особенно эти трудности усугубляются в бисингулярных задачах [1], когда коэффициенты разложений в пограничных слоях сами имеют особенности. Как правило, такая ситуация возникает в окрестностях угловых точек гранищы области и в окрестностях точек касания границы характеристиками предельного оператора.

Работа вьполнена при поддержке Российского фонда фундаментальных исследований (гранты № № 00-15-96038, 02-01-00768).

(C) А. Р. ДАнилин 2003 
Рассматриваемая задача служит модельньм примером, показывающим как возникающие затруднения, так и способы их преодоления. Отметим некоторые характерные отличия, возникающие при реализации известных общих подходов для построения полных асимптотических разложений в этом случае, по сравнению со скалярными задачами, порожденњыми аналогичными операторами в аналогичных областях (см. [7], [8]). Заметим, что эти особенности не позволяют дословно применить указанные там асимптотические процедуры.

Появление скалярного параметра с дополнительным интегральньм соотношением делает задачу нелинейной. При этом для определения коэффициентов разложения возникает необходимость нахождения асимптотики интегралов от произведения разномасштабных функций (заключительная часть $\S 3$ ).

Другая особенность - появление у внешнего разложения двух пограничных слоев в окрестности горизонтальных границ. При этом в силу “завязанности” системы уравнений в правых частях появляются функции, не характерные для соответствующих дифференциальных операторов из левой части. Отметим, что такая же ситуация возникает и во всех остальных пограничных областях, что требует модификации стандартных процедур нахождения следующих членов разложения.

Система уравнений, описывающая разложение вблизи вертикальных границ, не является ни эллиптической, ни параболической, ни гиперболической. При этом рассматриваемые задачи для произвольных систем такого вида, вообще говоря, не разрешимы (задача (1.18)).

В окрестностях угловых точек опять же в силу “завязанности” системы эллиптических задач для нахождения асимптотических разложений соответствующих коэффициентов возникает необходимость рассмотрения нетрадиционных для скалярного случая задач и введения новых классов функций для построения соответствующих анзацев (чему посвящена бо́льшая часть $\S 2$ ). В частности, в разложениях появляются логарифмические члены, отсутствующие в скалярном случае [7].

\section{§1. Постановка задачи и определяющие соотношения}

В области $\Omega:=(0 ; 1) \times(0 ; 1) \subset \mathbb{R}^{2}$ рассматривается следуюшая задача

$$
\begin{gathered}
\begin{cases}\mathscr{L}_{\varepsilon} z_{\varepsilon}+u_{\varepsilon}=f, \quad \mathscr{L}_{\varepsilon}^{*} u_{\varepsilon}-\lambda_{\varepsilon} z_{\varepsilon}=0, & (x, y) \in \Omega \\
z_{\varepsilon}(x, y)=u_{\varepsilon}(x, y)=0, & (x, y) \in \partial \Omega\end{cases} \\
\left\|u_{\varepsilon}\right\|^{2}:=\int_{\Omega} u_{\varepsilon}^{2}(x, y) d x d y=R^{2}
\end{gathered}
$$

где

$$
\begin{gathered}
\mathscr{L}_{\varepsilon} z:=\varepsilon^{2} \Delta z-b(x) \frac{\partial z}{\partial y}-a(x, y) z, \quad \mathscr{L}_{\varepsilon}^{*} u:=\varepsilon^{2} \Delta u+b(x) \frac{\partial u}{\partial y}-a(x, y) u, \\
f, a \in C^{\infty}(\bar{\Omega}), \quad a(x, y) \geqslant A>0 \\
b \in C^{\infty}[0 ; 1], \quad b(x) \geqslant B>0 \text { при } \quad(x, y) \in \bar{\Omega} \\
\text { при } x \in[0 ; 1]
\end{gathered}
$$

И

$$
b(x) \frac{\partial f(x, y)}{\partial y}-a(x, y) f(x, y) \not \equiv 0 \quad \text { в } \quad \Omega .
$$


Требуется построить равномерные асимптотики функций $z_{\varepsilon}, u_{\varepsilon}$ в области $\Omega$ и $\lambda_{\varepsilon}$ при $\varepsilon \rightarrow 0$.

К задаче (1.1), (1.2) сводится нахождение оптимального управления и соответствующего ему состояния в задаче оптимального управления с квадратичным критерием качества и ограничением на управление вида $\left\|u_{\varepsilon}\right\|^{2} \leqslant R^{2}[4]$.

В [4] показано, что справедливо следующее утверждение.

ТЕОРемА 1.1. Пусть выполнены условия (1.3) и (1.4) и для некоторого $\alpha>1$ функции $\bar{z}_{\varepsilon}, \bar{u}_{\varepsilon} \in C^{\infty}(\Omega)$ и $\bar{\lambda}_{\varepsilon}$ удовлетворяют следующим соотношениям

$$
\left\{\begin{array}{cc}
\mathscr{L}_{\varepsilon} \bar{z}_{\varepsilon}+\bar{u}_{\varepsilon}-f=o\left(\varepsilon^{\alpha}\right), \quad \mathscr{L}_{\varepsilon}^{*} \bar{u}_{\varepsilon}-\bar{\lambda}_{\varepsilon} \bar{z}_{\varepsilon}=o\left(\varepsilon^{\alpha}\right), & (x, y) \in \Omega \\
\bar{z}_{\varepsilon}(x, y)=o\left(\varepsilon^{\alpha}\right), \quad \bar{u}_{\varepsilon}(x, y)=o\left(\varepsilon^{\alpha}\right), & (x, y) \in \partial \Omega \\
\left\|\bar{u}_{\varepsilon}\right\|=R+o\left(\varepsilon^{\alpha}\right) &
\end{array}\right.
$$

при $\varepsilon \rightarrow 0$ в смысле метрики пространств $C^{2}(\Omega), C^{2}(\partial \Omega)$ соответственно, a $\bar{\lambda}_{\varepsilon}-\lambda_{0}=o(1), \bar{z}_{\varepsilon}(x)-z_{0}(x)=o(1), \bar{u}_{\varepsilon}(x)-u_{0}(x)=o(1)$ nрu $\varepsilon \rightarrow 0$ в $L_{2}(\Omega)$, әде $\lambda_{0}, z_{0}$ и $u_{0}$ - единственное решение задачи

$$
\begin{cases}\mathscr{L}_{0} z+u=f, & \mathscr{L}_{0}^{*} u-\lambda z=0 \\ z(x, 0)=0, & u(x, 1)=0,\|u\|=R .\end{cases}
$$

Тогда $\bar{\lambda}_{\varepsilon}-\bar{\lambda}_{\varepsilon}=o\left(\varepsilon^{\alpha}\right), \bar{z}_{\varepsilon}-z_{\varepsilon}=o\left(\varepsilon^{\alpha-1}\right), \bar{u}_{\varepsilon}-u_{\varepsilon}=o\left(\varepsilon^{\alpha-1}\right) n p u \varepsilon \rightarrow 0$ в $C(\bar{\Omega})$.

Эта теорема показывает, что для построения асимптотики функций $z_{\varepsilon}, u_{\varepsilon}$ и $\lambda_{\varepsilon}$ достаточно построить $\bar{z}_{\varepsilon}, \bar{u}_{\varepsilon}$ и $\bar{\lambda}_{\varepsilon}$, удовлетворяющие соотношениям (1.5), (1.6).

Будем искать эти функции методом согласования внешнего и внутреннего разложений [1].

Внешнее разложение определяется системой из (1.7). Однако в отличие от случая одного уравнения [1], [7] здесь пограничные слои возникают для обеих функций в окрестностях обеих горизонтальных границ ( $y=0$ и $y=1)$ (аналогичная ситуация возникала и в [9]).

Таким образом, внешнее разложение ищется в виде

$$
\left\{\begin{array}{l}
\underset{z}{\text { out }}=\sum_{k=0}^{\infty} \varepsilon^{k} \sum_{l=0}^{k-2}\left(z_{k, l}(x, y)+z_{k, l}^{-}\left(x, \eta_{-}\right)+z_{k, l}^{+}\left(x, \eta_{+}\right)\right) \ln ^{l} \varepsilon \\
u_{u}^{\text {out }}=\sum_{k=0}^{\infty} \varepsilon^{k} \sum_{l=0}^{k-2}\left(u_{k, l}(x, y)+u_{k, l}^{-}\left(x, \eta_{-}\right)+u_{k, l}^{+}\left(x, \eta_{+}\right)\right) \ln ^{l} \varepsilon \\
\bar{\lambda}_{\varepsilon}=\sum_{k=0}^{\infty} \varepsilon^{k} \sum_{l=0}^{k-2} \lambda_{k, l} \ln ^{l} \varepsilon
\end{array}\right.
$$

где $\eta_{-}:=y / \varepsilon^{2}, \eta_{+}:=(1-y) / \varepsilon^{2}$, a $z_{0,0}:=z_{0}, u_{0,0}:=u_{0}, \lambda_{0,0}:=\lambda_{0}$.

Подставим ряды (1.8) в систему (1.1) и приравняем члены одинакового вида и одного порядка малости.

Для функций $z_{k, l}(x, y) u_{k, l}(x, y)$ и $\lambda_{k, l}$ получим системы

$$
\left\{\begin{array}{l}
\mathscr{L}_{0} z_{k, l}+u_{k, l}=-\Delta z_{k-2, l} ; \\
\mathscr{L}_{0}^{*} u_{k, l}-\lambda_{0} z_{k, l}=\lambda_{k, l} z_{0}-\Delta z_{k-2, l}+g_{k, l},
\end{array}\right.
$$

где $g_{k, l}$ полностью определяются предыдушими уравнениями. 
Раскладывая $a(x, y)$ в ряды Тейлора в окрестности точек $y=0$ и $y=1$, получаем, что $z_{k, l}^{\mp}$ и $u_{k, l}^{\mp}$ удовлетворяют системам

$$
\left\{\begin{array}{c}
\mathscr{M}^{\mp}\left(x, \eta_{\mp}\right) z_{k, l}^{\mp}=f_{k, l}^{\mp}\left(x, \eta_{\mp}\right) ; \\
\mathscr{M}^{ \pm}\left(x, \eta_{\mp}\right) u_{k, l}^{\mp}=g_{k, l}^{\mp}\left(x, \eta_{\mp}\right),
\end{array}\right.
$$

где $\mathscr{M}^{\mp}(x, \eta):=\partial^{2} / \partial \eta \mp b(x) \partial / \partial \eta$, a $f_{k, l}^{\mp}, g_{k, l}^{\mp}$ полностью определяются предыдущими уравнениями.

Все системы $(1.9),(1.10)$ связаны общими условиями на границах $y=0$ и $y=1$ и поведением при $\eta_{\mp} \rightarrow \infty$ :

$$
\left\{\begin{array}{c}
z_{k, l}(x, 0)+z_{k, l}^{-}(x, 0)=0=u_{k, l}(x, 0)+u_{k, l}^{-}(x, 0) ; \\
z_{k, l}(x, 1)+z_{k, l}^{+}(x, 0)=0=u_{k, l}(x, 1)+u_{k, l}^{+}(x, 0),
\end{array}\right.
$$

$z_{k, l}^{\mp}$ и $u_{k, l}^{\mp}$ экспоненциально убывают при $\eta_{\mp} \rightarrow \infty$ равномерно по $x \in[0 ; 1]$.

ТЕОРЕМА 1.2. При фиксированном наборе $\left\{\lambda_{k, l}\right\}$ существуют однозначно определенные решения системы (1.9), (1.10), удовлетворяющие условия.м (1.11), (1.12). При этом все функиии являются гладкими в соответствующих замкнутых областях, а функции погранслоев имеют следующий вид:

$$
\begin{aligned}
& z_{k, l}^{\mp}\left(x, \eta_{\mp}\right)=P_{k, l}^{\mp}\left(\eta_{\mp} ; n_{k, l}^{\mp} ; x\right) \exp \left(-b(x) \eta_{\mp}\right), \\
& u_{k, l}^{\mp}\left(x, \eta_{\mp}\right)=Q_{k, l}^{\mp}\left(\eta_{\mp} ; m_{k, l}^{\mp} ; x\right) \exp \left(-b(x) \eta_{\mp}\right),
\end{aligned}
$$

әде $n_{k, l}^{-}:=m_{k, l}^{+}:=[(k-l-2) / 2], n_{k, l}^{+}:=m_{k, l}^{-}:=n_{k, l}^{-}+1,[\cdot]-$ челая часть числа, a $P(\eta ; n ; x), Q(\eta ; n ; x)$ - многочлены по переменной $\eta$ степени $n$ с коэффициентами, гладко зависящими от $x$ (как обьчно, считаем, что многочлень отрицательной степени тожсдественно равнь нулю).

ДокАЗАТЕЛЬство. Прежде всего отметим, что все системы уравнений - это системы обыкновенных дифференциальных уравнений с гладкими коэффициентами, гладко зависящими от параметра $x$. Поэтому и их решения, если они сушествуют, являются гладкими функциями своих аргументов.

Определение функций $z_{k, l}^{(\mp)}$ и $u_{k, l}^{(\mp)} 1$ при фиксированных $\lambda_{k, l}$ происходит следующим образом. Сначала однозначно определяются $z_{k, l}^{-}$и $u_{k, l}^{+}$из условия экспоненциального убывания при $\eta_{\mp} \rightarrow+\infty$. Функции $z_{k, l}^{-}$и $u_{k, l}^{+}$в силу (1.11) определяют начальные условия $z_{k, l}(x, 0)$ и $u_{k, l}(x, 1)$, по которым находятся $z_{k, l}$ и $u_{k, l}$. И, наконец, определяются начальные условия $z_{k, l}^{+}(x, 0)=-z_{k, l}(x, 1)$ и $u_{k, l}^{-}(x, 0)=$ $-u_{k, l}(x, 0)$, что позволяет определить оставшиеся функции.

Проведя индукцию по $(k, l)$, получим (1.13).

\footnotetext{
${ }^{1}$ Запись вида $W^{(s)}$ здесь и в дальнейшем исползуется как общее обозначение для $W$ и $W^{s}$.
} 
ТЕОРема 1.3. Пусть $\widehat{z}_{N}, \widehat{u}_{N}$ и $\widehat{\lambda}_{N}-$ частичные суммы рядов (1.8). Тогда найдется $\gamma>0$ maкое, что

$$
\begin{gathered}
\mathscr{L}_{\varepsilon} \widehat{z}_{N}+\widehat{u}_{N}=f+o\left(\varepsilon^{N}\right), \quad \mathscr{L}_{\varepsilon}^{*} \widehat{u}_{N}+\widehat{\lambda}_{N} \widehat{z}_{N}=o\left(\varepsilon^{N}\right), \quad(x, y) \in \bar{\Omega}, \\
\widehat{z}_{N}=O\left(e^{-\gamma / \varepsilon^{2}}\right), \quad \widehat{u}_{N}=O\left(e^{-\gamma / \varepsilon^{2}}\right), \quad(x, y) \in[0 ; 1] \times\{0 ; 1\} .
\end{gathered}
$$

ДоКАЗАТЕЛЬСТво непосредственно следует из определения $\widehat{z}_{N}, \widehat{u}_{N}$ и $\widehat{\lambda}_{N}$ и гладкости $z_{k, l}^{(\mp)}$ и $u_{k, l}^{(\mp)}$ в соответствуюших областях.

Отметим, что граничные условия при $x=0$ и $x=1$, вообше говоря, не выполнены.

В окрестности границ $x=0$ и $x=1$ асимптотическое разложение будем искать в виде рядов функций, зависящих от новых "растянутых" переменных.

Рассмотрим подробнее окрестность границы $x=0$. Здесь новая переменная $\zeta:=x / \varepsilon$.

Функции $Z_{\varepsilon}(\zeta, y):=z_{\varepsilon}(\varepsilon \zeta, y)$ и $U_{\varepsilon}(\zeta, y):=u_{\varepsilon}(\varepsilon \zeta, y)$ в области $\Pi:=(0 ; \infty) \times(0 ; 1)$ удовлетворяют системе

$$
\left\{\begin{array}{l}
\frac{\partial^{2} Z_{\varepsilon}}{\partial \zeta^{2}}-b(\varepsilon \zeta) \frac{\partial Z_{\varepsilon}}{\partial y}-a(\varepsilon \zeta, y) Z_{\varepsilon}+U_{\varepsilon}+\varepsilon^{2} \frac{\partial^{2} Z_{\varepsilon}}{\partial y^{2}}=f(\varepsilon \zeta, y) \\
\frac{\partial^{2} U_{\varepsilon}}{\partial \zeta^{2}}+b(\varepsilon \zeta) \frac{\partial U_{\varepsilon}}{\partial y}-a(\varepsilon \zeta, y) U_{\varepsilon}-\bar{\lambda}_{\varepsilon} Z_{\varepsilon}+\varepsilon^{2} \frac{\partial^{2} U_{\varepsilon}}{\partial y^{2}}=0 \\
Z_{\varepsilon}(\zeta, y)=0=U_{\varepsilon}(\zeta, y), \quad(\zeta, y) \in \partial \Pi
\end{array}\right.
$$

Для удовлетворения граничных условий и здесь необходимо рассмотреть пограничные слои и соответствуюшие им погранфункции. Таким образом, внутреннее разложение тоже ищем в виде

$$
\begin{aligned}
& \stackrel{\text { in }}{Z}=\sum_{k=0}^{\infty} \varepsilon^{k} \sum_{l=0}^{k-2}\left(Z_{k, l}(\zeta, y)+Z_{k, l}^{-}\left(\zeta, \eta_{-}\right)+Z_{k, l}^{+}\left(\zeta, \eta_{+}\right)\right) \ln ^{l} \varepsilon, \\
& \stackrel{\text { in }}{U}=\sum_{k=0}^{\infty} \varepsilon^{k} \sum_{l=0}^{k-2}\left(U_{k, l}(\zeta, y)+U_{k, l}^{-}\left(\zeta, \eta_{-}\right)+U_{k, l}^{+}\left(\zeta, \eta_{+}\right)\right) \ln ^{l} \varepsilon .
\end{aligned}
$$

Здесь снова, как и во внешнем разложении, $\eta_{-}:=y / \varepsilon^{2}$, а $\eta_{+}:=(1-y) / \varepsilon^{2}$.

Внутреннее разложение при $\zeta \rightarrow+\infty$ должно быть согласовано с внешним при $x \rightarrow 0$.

Перераскладывая внешшее разложение при $x \rightarrow 0$ и делая замену $x=\varepsilon \zeta$, получаем

$$
\begin{aligned}
& \underset{z}{\operatorname{out}}(\varepsilon \zeta, y)=\sum_{k=0}^{\infty} \varepsilon^{k} \sum_{l=0}^{k-2}\left(1_{k, l}(\zeta, y)+\stackrel{1}{Z}_{k, l}^{-}\left(\zeta, \eta_{-}\right)+\stackrel{1}{Z}_{k, l}^{+}\left(\zeta, \eta_{+}\right)\right) \ln ^{l} \varepsilon \\
& \stackrel{\text { out }_{u}}{(\varepsilon \zeta, y)}=\sum_{k=0}^{\infty} \varepsilon^{k} \sum_{l=0}^{k-2}\left(\stackrel{1}{U}_{k, l}(\zeta, y)+\stackrel{1}{U}_{k, l}^{-}\left(\zeta, \eta_{-}\right)+\stackrel{1}{U}_{k, l}^{+}\left(\zeta, \eta_{+}\right)\right) \ln ^{l} \varepsilon
\end{aligned}
$$


где

$$
\left\{\begin{array}{l}
\stackrel{1}{Z}_{k, 0}(\zeta, y)=P_{k, 0}(\zeta ; k ; y) \\
\stackrel{1}{Z}_{k, 0}^{-}\left(\zeta, \eta_{-}\right)=\exp \left(-\eta_{-} b_{0}\right) \sum_{s=0}^{k-2} \zeta^{s} P_{k, 0, s}^{-}\left(\eta_{-} ; s\right) \\
\stackrel{1}{Z}_{k, l}^{(-)} \sim \stackrel{1}{Z}_{k-l-2,0}^{(-)}, \quad l>0 \\
\stackrel{1}{U_{k, l}^{-}} \sim \stackrel{1}{Z}_{k, l}^{+}, \quad \stackrel{1}{U_{k, l}^{+}} \sim \stackrel{1}{Z_{k, l}^{-}}, \quad \stackrel{1}{U_{k, l}^{-}} \sim \stackrel{1}{Z}_{k+2, l}^{-} .
\end{array}\right.
$$

Здесь $P(\eta ; m)$ - многочлены степени $m$ от $\eta, b_{0}:=b(0)$, символ $\sim$ означает "имеет аналогичньй вид".

Не ограничивая общности, можно считать, что $b_{0}=1$.

Так как все суммы, которыми определяются ${ }_{k, l}^{(\mp)}$ и $\stackrel{1}{U}_{k, l}^{(\mp)}$, конечны, то эти фуункции удовлетворяют системе, полученной из (1.14) переразложением при $\varepsilon \zeta \rightarrow 0$.

Функции $Z_{k, l}^{(\mp)}, U_{k, l}^{(\mp)}$ будем искать в виде

$$
Z_{k, l}^{(\mp)}=\stackrel{1}{Z}_{k, l}^{(\mp)}+\stackrel{2}{Z}{ }_{k, l}^{(\mp)}, \quad U_{k, l}^{(\mp)}=\stackrel{1}{U}_{k, l}^{(\mp)}+\stackrel{2}{U_{k, l}^{(\mp)}}
$$

Тогда $\stackrel{2}{Z}_{k, l}^{(\mp)}, \stackrel{2}{U}(\mp)$ являются решением системы

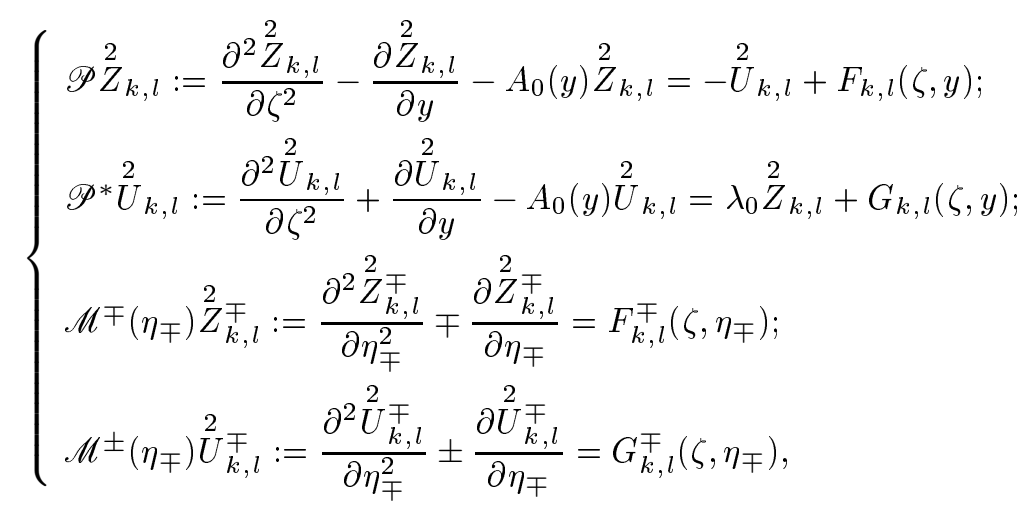

где $F_{k, l}(\zeta, y), G_{k, l}(\zeta, y), F_{k, l}^{\mp}\left(\zeta, \eta_{\mp}\right)$ и $G_{k, l}^{\mp}\left(\zeta, \eta_{\mp}\right)$ полностью определяются решениями предыдущих уравнений с помощью операторов дифференцирования первого и второго порядков по переменным $у$ и $\zeta$.

Все системы связаны общими условиями

$$
\left\{\begin{aligned}
0 & =\stackrel{2}{Z}_{k, l}^{(\mp)}(0, y)+\stackrel{1}{Z}_{k, l}^{(\mp)}(0, y)=\stackrel{2}{U_{k, l}^{(\mp)}}(0, y)+\stackrel{1}{U}_{k, l}^{(\mp)}(0, y) \\
0 & \stackrel{2}{Z_{k, l}}(\zeta, 0)+\stackrel{2}{Z}_{k, l}^{-}(\zeta, 0)=\stackrel{2}{Z}_{k, l}(\zeta, 1)+\stackrel{2}{Z}_{k, l}^{+}(\zeta, 0) \\
& \stackrel{2}{2} \stackrel{2}{U}_{k, l}^{-}(\zeta, 0)+\stackrel{2}{U}_{k, l}^{2}(\zeta, 0)=\stackrel{U}{U}_{k, l}^{+}(\zeta, 1)+(\zeta, 0)
\end{aligned}\right.
$$

Отметим, что уравнения для $\stackrel{2}{Z}_{k, l}^{\mp}$ и $\stackrel{2}{U}_{k, l}^{\mp}$ - это снова обыкновенные дифференциальные уравнения, зависящие от параметра $\zeta$. 
Для согласования внешнего и внутреннего разложений требуется, чтобы $\stackrel{2}{Z}_{k, l}^{\mp}$ и $\stackrel{2}{U}_{k, l}^{\mp}$ убывали при $\zeta \rightarrow+\infty$ быстрее любой степени $\zeta$.

Рассмотрим подробнее стандартную задачу для $\stackrel{2}{Z} \underset{k, l}{ } \stackrel{2}{{ }^{\prime}} \underset{k, l}{ }$ :

$$
\left\{\begin{array}{l}
\mathscr{P} Z+U=F(\zeta, y), \mathscr{P} * U-\lambda_{0} Z=G(\zeta, y), \quad(\zeta, y) \in \Pi ; \\
Z(0, y)=\varphi(y), U(0, y)=\psi(y), \quad y \in[0 ; 1] ; \\
Z(\zeta, 0)=\Phi(\zeta), \quad U(\zeta, 1)=\Psi(\zeta) ; \\
F(\zeta, y), G(\zeta, y), \Phi(\zeta), \Psi(\zeta) \quad \text { суть } O\left(e^{-\gamma \zeta}\right) \text { при } \zeta \rightarrow+\infty \\
\quad \text { вместе с производнњми по } \zeta \text { до второго порядка. }
\end{array}\right.
$$

Не ограничивая общности (сделав замену $\bar{Z}=Z+\Phi, \bar{U}=U+\Psi$ ), можно считать, что $\Phi(\zeta) \equiv \Psi(\zeta) \equiv 0$.

Пусть $Z_{0}$ и $U_{0}$ - решение задачи

$$
\left\{\begin{array}{l}
\frac{\partial^{2} Z_{0}}{\partial \zeta^{2}}=\frac{\partial Z_{0}}{\partial y}, \quad Z_{0}(0, y)=\varphi(y), \quad Z_{0}(\zeta, 0)=0 \\
\frac{\partial^{2} U_{0}}{\partial \zeta^{2}}=-\frac{\partial U_{0}}{\partial y}, \quad U_{0}(0, y)=\psi(y), \quad U_{0}(\zeta, 1)=0 .
\end{array}\right.
$$

Известно [15; гл. III], что если $\varphi(0)=0=\psi(1)$, то $Z_{0}, U_{0} \in C^{\infty}(\Pi) \cap C^{1}(\bar{\Pi})$ и

$$
\left|Z_{0}\right|,\left|U_{0}\right| \leqslant K\left(\|\varphi\|_{C[0 ; 1]}+\|\psi\|_{C[0 ; 1]}\right) e^{-\zeta^{2} / 8}
$$

Для $W:=Z-Z_{0}, V:=U-U_{0}$ получим следуюшую систему

$$
\left\{\begin{array}{l}
\mathscr{P} W+V=F_{1}, \mathscr{P}^{*} V-\lambda_{0} W=G_{1}, \quad(\zeta, y) \in \Pi ; \\
0=W(0, y)=V(0, y)=W(\zeta, 0)=V(\zeta, 1)
\end{array}\right.
$$

где $F_{1}, G_{1} \in C^{\infty}(\Pi) \cap C^{1}(\bar{\Pi})$ и $\left|F_{1}\right|,\left|G_{1}\right| \leqslant K_{1} e^{-\gamma \zeta}$.

Справедлива следуюшая теорема [17].

Теорема 1.4. Задача (1.20) разрешима единственныцм образом в

$$
C_{\delta}(\bar{\Pi}):=\left\{Z(\zeta, y) \in C(\bar{\Pi}): \sup _{\zeta \geqslant 0} \max _{0 \leqslant y \leqslant 1}\left|e^{\delta \zeta} Z(\zeta, y)\right|<+\infty\right\}
$$

при $\delta<\delta_{0}:=\min \{\gamma, \sqrt{A}\} / 2$, и справедливъ следующие неравенства

$$
\begin{gathered}
|W(\zeta, y)| \leqslant K\left(\lambda_{0}, \delta_{0}, A\right)\left(\left\|e^{\delta_{0} \zeta} F_{1}\right\|+\left\|e^{\delta_{0} \zeta} G_{1}\right\|\right) e^{-2 \delta_{0} \zeta}, \\
|V(\zeta, y)| \leqslant K\left(\lambda_{0}, \delta_{0}, A\right)\left(\left\|e^{\delta_{0} \zeta} F_{1}\right\|+\left\|e^{\delta_{0} \zeta} G_{1}\right\|\right) e^{-2 \delta_{0} \zeta} .
\end{gathered}
$$

Отметим, что уже решение системы (1.19) имеет особенности производных в угловых точках. Тем более это относится к решению системы (1.20).

В связи с этим $\stackrel{2}{Z}_{k, l}$ и $\stackrel{2}{U}_{k, l}$ имеют нарастающие особенности в угловых точках, и без информации о характере этих особенностей нужные нам решения из (1.16) однозначно не определить. Для уяснения этих особенностей необходимо рассмотреть угловые точки $(0 ; 0)$ и $(0 ; 1)$. 
Поскольку решения системы $(1.1)$ в окрестностях точек $(0 ; 0)$ и $(0 ; 1)$ ведут себя сходным образом, то рассмотрим подробнее точку $(0 ; 0)$.

Замена $\xi:=x / \varepsilon^{2}, \eta:=y / \varepsilon^{2}$ для функций

$$
w_{\varepsilon}^{-}(\xi, \eta):=z_{\varepsilon}\left(\varepsilon^{2} \xi, \varepsilon^{2} \eta\right), \quad v_{\varepsilon}^{-}(\xi, \eta):=u_{\varepsilon}\left(\varepsilon^{2} \xi, \varepsilon^{2} \eta\right)
$$

в области $\omega:=(0 ; \infty) \times(0 ; \infty)$ приводит к задаче

$$
\left\{\begin{array}{l}
\Delta w_{\varepsilon}^{-}-b\left(\varepsilon^{2} \xi\right) \frac{\partial w_{\varepsilon}^{-}}{\partial \eta}-\varepsilon^{2} a\left(\varepsilon^{2} \xi, \varepsilon^{2} \eta\right) w_{\varepsilon}^{-}+\varepsilon^{2} v_{\varepsilon}^{-} \\
=\varepsilon^{2} f\left(\varepsilon^{2} \xi, \varepsilon^{2} \eta\right), \quad(\xi, \eta) \in \omega ; \\
\Delta v_{\varepsilon}^{-}+b\left(\varepsilon^{2} \xi\right) \frac{\partial v_{\varepsilon}^{-}}{\partial \eta}-\varepsilon^{2} a\left(\varepsilon^{2} \xi, \varepsilon^{2} \eta_{-}\right) v_{\varepsilon}^{-}-\varepsilon^{2} \bar{\lambda}_{\varepsilon} w_{\varepsilon}^{-}=0 \\
0=w_{\varepsilon}^{-}(\xi, \eta)=v_{\varepsilon}^{-}(\xi, \eta), \quad(\xi, \eta) \in \partial \omega .
\end{array}\right.
$$

Асимптотику функций $w_{\varepsilon}^{-}, v_{\varepsilon}^{-}$ищем в виде

$$
w^{-}=\sum_{k=0}^{\infty} \varepsilon^{k} \sum_{l=0}^{k-2} w_{k, l}^{-} \ln ^{l} \varepsilon, \quad v^{-}=\sum_{k=0}^{\infty} \varepsilon^{k} \sum_{l=0}^{k-2} v_{k, l}^{-} \ln ^{l} \varepsilon .
$$

Тогда для функций $w_{k, l}^{-}$и $v_{k, l}^{-}$получим задачи

$$
\left\{\begin{array}{l}
L^{-} w_{k, l}^{-}=p_{k, l}^{-}(\xi, \eta), L^{+} v_{k, l}^{-}=q_{k, l}^{-}(\xi, \eta), \quad(\xi, \eta) \in \omega ; \\
w_{k, l}^{-}(\xi, \eta)=v_{k, l}^{-}(\xi, \eta)=0, \quad(\xi, \eta) \in \partial \omega .
\end{array}\right.
$$

Здесь $L^{\mp}:=\Delta \mp \partial / \partial \eta$, а

$$
\begin{aligned}
& p_{k, l}^{-}=\sum_{s=1} b_{s} \xi^{s} \frac{\partial}{\partial \eta} w_{k-2 s, l}^{-}+\sum_{s=0} A_{s}(\xi, \eta) w_{k-2-2 s, l}^{-}-v_{k-2, l}^{-}+f_{k}^{-}, \\
& q_{k, l}^{-}=-\sum_{s=1} b_{s} \xi^{s} \frac{\partial}{\partial \eta} v_{k-2 s, l}^{-}+\sum_{s=0} A_{s}(\xi, \eta) v_{k-2-2 s, l}^{-}+\sum_{s, \sigma=0} \lambda_{s, \sigma} w_{k-2-s, l-\sigma}^{-},
\end{aligned}
$$

$\left\{b_{s}\right\}$ - коэффициенты ряда Тейлора функции $b(x)$ при $x \rightarrow 0$,

$\left\{A_{s}(\xi, \eta)\right\}$ - однородные многочлены соответствуюшей степени, получаюшиеся при разложении функции $a(x, y)$ в окрестности точки $(0 ; 0)$,

$\left\{f_{2 m}^{-}\right\}$- однородные многочлены степени $m$, получающиеся при разложении функции $f(x, y)$ в окрестности точки $(0 ; 0)$, а $f_{2 m+1}^{-} \equiv 0$.

Известно [15; гл. VII], что задача

$$
L^{-} w(\xi, \eta)=p(\xi, \eta), \quad(\xi, \eta) \in \omega, \quad w(\xi, \eta)=0, \quad(\xi, \eta) \in \partial \omega,
$$

имеет единственное решение в классе медленно растуших функций, если такова и функция $p$. Решение этой задачи имеет вид

где

$$
w(\xi, \eta)=\frac{1}{2 \pi} \int_{0}^{\infty} \int_{0}^{\infty} \mathscr{K}(\xi, \eta, \bar{\xi}, \bar{\eta}) e^{(\eta-\bar{\eta}) / 2} p(\bar{\xi}, \bar{\eta}) d \bar{\xi} d \bar{\eta}
$$

$$
\begin{aligned}
\mathscr{K}(\xi, \eta, \bar{\xi}, \bar{\eta})= & -K_{0}\left(\frac{1}{2} \sqrt{(\xi-\bar{\xi})^{2}+(\eta-\bar{\eta})^{2}}\right)+K_{0}\left(\frac{1}{2} \sqrt{(\xi-\bar{\xi})^{2}+(\eta+\bar{\eta})^{2}}\right) \\
& +K_{0}\left(\frac{1}{2} \sqrt{(\xi+\bar{\xi})^{2}+(\eta-\bar{\eta})^{2}}\right)-K_{0}\left(\frac{1}{2} \sqrt{(\xi+\bar{\xi})^{2}+(\eta+\bar{\eta})^{2}}\right),
\end{aligned}
$$

a $K_{0}(\cdot)$ - функция Макдональда [16] и $w \in C^{1}(\bar{\omega}) \cap C^{\infty}\left(\omega^{\prime}\right), \omega^{\prime}:=\bar{\omega} \backslash\{(0 ; 0)\}$.

Вторая стандартная задача из (1.23) устроена хуже. Она имеет единственное решение лишь в классе ограниченных функций. 
Лемма 1.1. Задача

$$
L^{+} v(\xi, \eta)=0, \quad(\xi, \eta) \in \omega, \quad v(\xi, \eta)=0, \quad(\xi, \eta) \in \partial \omega
$$

в классе умеренно растущих функиий имеет бесконечно много решений. При этом для любого многочлена $P(\eta ; k)$ существуют однозначно определенные многочлены

$$
\begin{aligned}
& Q(\xi, \eta)=\xi Q_{1}(\eta ; k)+\xi^{3} Q_{3}(\eta ; k-1)+\cdots+\xi^{2 k+1} Q_{2 k+1}(\eta ; 0), \\
& R(\xi, \eta)=\xi R_{1}(\eta ; k)+\xi^{3} R_{3}(\eta ; k-1)+\cdots+\xi^{2 k+1} R_{2 k+1}(\eta ; 0)
\end{aligned}
$$

такие, что $v(\xi, \eta):=Q(\xi, \eta)+R(\xi, \eta) e^{-\eta}$ есть решение задачи (1.25). При әтом $Q_{1}(\eta ; k)=P(\eta ; k)$.

Обратно, если $Q(\xi, \eta)$ и $R(\xi, \eta)$ - многочлены и $Q+R e^{-\eta}$ - решение задачи (1.25), то $P$ и $Q$ имеют указанный вид.

ДокАЗАТЕЛЬСТво этой леммы получается простьм вычислением.

Отметим, что если функция $q(\xi, \eta)$ ограничена и абсолютно интегрируема в $\omega$, то единственное ограниченное решение задачи

$$
L^{+} v(\xi, \eta)=q(\xi, \eta), \quad(\xi, \eta) \in \omega, \quad v(\xi, \eta)=0, \quad(\xi, \eta) \in \partial \omega
$$

находится по формуле (см., например, [1])

$$
v(\xi, \eta)=\frac{1}{2 \pi} \int_{0}^{\infty} \int_{0}^{\infty} \mathscr{K}(\xi, \eta, \bar{\xi}, \bar{\eta}) e^{(\bar{\eta}-\eta) / 2} q(\bar{\xi}, \bar{\eta}) d \bar{\xi} d \bar{\eta}
$$

Решения же задач

$$
\begin{aligned}
& L^{-} w(\xi, \eta)=0, \quad(\xi, \eta) \in \omega, \quad w(0, \eta)=\varphi(\eta), \quad w(\xi, 0)=0, \\
& L^{+} v(\xi, \eta)=0, \quad(\xi, \eta) \in \omega, \quad v(0, \eta)=\psi(\eta), \quad v(\xi, 0)=\chi(\xi),
\end{aligned}
$$

при медленно растушей $\varphi$ и абсолютно интегрируемых $\psi$ и $\chi$ находятся по формулам (см., например, [1])

$$
\begin{aligned}
w(\xi, \eta)= & -\frac{1}{2 \pi} \int_{0}^{\infty} \frac{\partial}{\partial \bar{\xi}} \mathscr{K}(\xi, \eta, 0, \bar{\eta}) e^{(\eta-\bar{\eta}) / 2} \varphi(\bar{\eta}) d \bar{\eta} \\
v(\xi, \eta)= & -\frac{1}{2 \pi} \int_{0}^{\infty} \frac{\partial}{\partial \bar{\xi}} \mathscr{K}(\xi, \eta, 0, \bar{\eta}) e^{(\bar{\eta}-\eta) / 2} \psi(\bar{\eta}) d \bar{\eta} \\
& -\frac{1}{2 \pi} \int_{0}^{\infty}\left(\frac{\partial}{\partial \bar{\eta}} \mathscr{K}(\xi, \eta, \bar{\xi}, 0)+\frac{\mathscr{K}(\xi, \eta, \bar{\xi}, 0)}{2}\right) e^{-\eta / 2} \chi(\bar{\xi}) d \bar{\xi}
\end{aligned}
$$

Формулы (1.24), (1.26), (1.29) и (1.30) позволяют получить утверждения о поведении решений при $\xi, \eta \rightarrow+\infty$, если известны поведения функций $p, q, \varphi, \psi$ и $\chi$. 
ЛЕмма 1.2. Пусть $p \in C(\bar{\omega}) \cap C^{\infty}\left(\omega^{\prime}\right), \varphi \in C^{\infty}[0 ; \infty)$,

$$
\begin{aligned}
\left|D^{k} \varphi(\eta)\right| \leqslant M_{k} \eta^{-N} \quad \text { nрu } \quad \eta>0, \\
\left|D^{k} p(\xi, \eta)\right| \leqslant M_{k} \eta^{-N} \quad \text { в } \quad \omega, \\
\left|D^{k} p(\xi, \eta)\right| \leqslant M_{k} \xi^{-N} \quad \text { nрu } \quad \xi \geqslant E \eta+1 .
\end{aligned}
$$

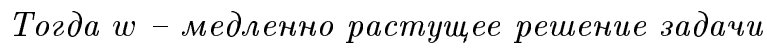

$$
L^{-} w(\xi, \eta)=p(\xi, \eta), \quad(\xi, \eta) \in \omega, \quad w(0, \eta)=\varphi(\eta), \quad w(\xi, 0)=0,
$$

удовлетворяет следующим соотношениям:

$$
\begin{gathered}
\left|D^{k} w\right| \leqslant \bar{M}_{k} \xi^{-N+2} \quad \text { npu } \quad \xi \geqslant E \eta+1, \quad \xi^{2}+\eta^{2} \geqslant 1, \\
D^{k} w=D^{k}\left(\sum_{s=0}^{N_{1}} \eta^{-(s+1) / 2} \Phi_{s}(\theta)+O\left((\eta+\xi)^{-N_{1}}\right)\right),
\end{gathered}
$$

əде $N_{1} \rightarrow+\infty$ при $N \rightarrow+\infty, \theta=\xi /(2 \sqrt{\eta}), a \Phi_{s} \in C^{\infty}[0 ; \infty],\left|\Phi_{s}(\theta)\right| \leqslant K_{s} e^{-\bar{\gamma} \theta^{2}}$.

ДокАЗАТЕльство аналогично доказательству леммы 1.4 из [1;гл. IV] при этом область интегрирования разбивается на следующие части:

$$
\begin{gathered}
\mathscr{G}_{1}: \bar{\xi}^{2}+\bar{\eta}^{2} \leqslant 1 ; \quad \mathscr{G}_{2}: \bar{\xi}^{2}+\bar{\eta}^{2}>1, \quad \bar{\eta} \leqslant \frac{2 \xi}{E}, \quad \bar{\xi} \leqslant \frac{\xi}{2} \\
\mathscr{G}_{3}: \bar{\eta} \leqslant \frac{2 \xi}{E}, \quad \bar{\xi}>\frac{\xi}{2} ; \quad \mathscr{G}_{4}: \bar{\eta}>\frac{2 \xi}{E} .
\end{gathered}
$$

В дальнейшем функции $w$, удовлетворяющие неравенствам

$$
\left|D^{k} w\right| \leqslant \bar{M}_{k} e^{-\gamma \xi} \text { при } \xi \geqslant E \eta+1, \quad \xi^{2}+\eta^{2} \geqslant 1
$$

и имеющие при $\eta \rightarrow \infty$ асимптотику вида $w=\sum_{s=0}^{\infty} \eta^{(m-s) / 2} \Phi_{s}(\theta)$, будем обозначать через $\Xi(\xi, \eta ; m)$.

Лемма 1.3. Пусть $q \in C^{\infty}\left(\omega^{\prime}\right), \psi \in C^{\infty}[0 ; \infty)$,

$$
\begin{aligned}
&\left|D^{k} \psi(\eta)\right| \leqslant M_{k} \eta^{-N} \text { nрu } \quad \eta>0, \\
&\left|D^{k} q(\xi, \eta)\right| \leqslant M_{k} \eta^{-N} \quad \text { в } \quad \omega, \\
&\left|D^{k} q(\xi, \eta)\right| \leqslant M_{k} \xi^{-N} \quad \text { nрu } \quad \xi \geqslant E \eta .
\end{aligned}
$$

Тогда $v-$-решение задачи

$$
L^{+} v(\xi, \eta)=q(\xi, \eta), \quad(\xi, \eta) \in \omega, \quad v(0, \eta)=\psi(\eta), \quad v(\xi, 0)=0,
$$

найденное по формулам (1.26), (1.30), удовлетворяет следующим соотношениям:

$$
\begin{array}{rlll}
\left|D^{k} v(\xi, \eta)\right| \leqslant M_{k} \xi^{-N+2} & \text { npu } & \xi \geqslant E \eta, & \xi^{2}+\eta^{2} \geqslant 1 \\
\left|D^{k} v\right|=O\left(\eta^{-N+2}\right) & \text { nрu } & \eta \rightarrow+\infty
\end{array}
$$


ДОКАЗАТЕЛЬСТВО оПять проводится с помошью оценки соответствующих интегралов при разбиении области интегрирования на части

$$
\mathscr{G}_{1}: \bar{\xi}, \bar{\eta} \leqslant \frac{\eta}{2} ; \quad \mathscr{G}_{2}: \bar{\eta}>\frac{\eta}{2}, \quad \bar{\eta} \geqslant \bar{\xi} ; \quad \mathscr{G}_{3}: \bar{\xi} \geqslant \frac{\eta}{2}, \quad \bar{\eta} \leqslant \bar{\xi} .
$$

Аналогично, из явного вида решений доказывается и следуюшая лемма.

Лемма 1.4. Пусть $\chi(\xi) \in C^{\infty}[0 ; \infty) u$

$$
\left|D^{k} \chi(\xi)\right| \leqslant M_{k} \xi^{-N} \quad \text { в } \quad \omega .
$$

Тогда $v$ - решение задачи (1.28) с $\psi \equiv 0$, найденное по формуле (1.30), удовлетворяет неравенствам

$$
\left|D^{k} v(\xi, \eta)\right| \leqslant M_{k} e^{-\eta / 2} \xi^{-N+1} \quad \text { npu } \quad \eta^{2}+\xi^{2} \geqslant 1 .
$$

Для выяснения вида решений задач (1.23) и их асимптотики при $\xi, \eta \rightarrow \infty$ удобно рассмотреть некоторые классы функций, в которых соответствующие задачи разрешимы. Функции из этих классов позволяют построить ф.а.р. (формальное асимптотическое разложение) задач (1.23), а доказанные оценки - обосновать наличие решений с требуемьм видом асимптотики.

\section{§2. Вид решений внутренних задач}

Первый класс функций, который мы рассмотрим, имеет вид $P+Q e^{-\eta}$, где $P$ и $Q$ - многочлены переменных $\xi, \eta$.

Под параболической степенью одночлена $\xi^{k} \eta^{n}$ будем понимать число $k+2 n$, после чего параболическая степень многочлена определяется стандартно.

Тот факт, что многочлен $P$ имеет степень $k$ по $\xi$ и параболическую степень, равную $m$, будем записывать следующим образом $P(\xi, \eta ;(m, k))$. Однородньй в смысле параболической степени многочлен $P$ параболической степени $m$ будем записывать в виде $P(\xi, \eta ;(m, o))$.

Непосредственным вычислением показывается справедливость следуюшей леммы.

Лемма 2.1. 1) Задача $L^{+} v(\xi, \eta)=P(\xi, \eta ;(m, k)), v(0, \eta)=0$ имеет решение вида $v=R(\xi, \eta ;(m+2, m+2))$.

2) Задача $L^{-} w(\xi, \eta)=P(\xi, \eta ;(m, k)), w(\xi, 0)=0$ имеет решение вида $w=$ $R(\xi, \eta ;(m+2, k))$.

3) Задача $L^{-} w(\xi, \eta)=0, w(\xi, 0)=P(\xi, k)$ имеет решение вида $w=$ $R(\xi, \eta ;(k, k))$ u $w(0, \eta)=Q(\eta, ;[k / 2])$.

Следствием этой леммы и леммы 1.4 из [1; гл. IV] является

Tеорема 2.1. Задача $L^{-} w(\xi, \eta)=P(\xi, \eta ;(m, k)), w(\xi, 0)=0, w(0, \eta)=0$, имеет решение вида $w=R(\xi, \eta ;(m+2, k))+\Xi(\xi, \eta ; 2+2[m / 2])$.

Лемма 2.2. Задача $L^{+} v(\xi, \eta)=0, v(0, \eta)=P(\eta ; k), v(\xi, 0)=0$, имеет решение вида $v=R(\xi, \eta ;(2 k, 2 k))+S(\xi, \eta ;(2 k, 2 k)) e^{-\eta}+\stackrel{e}{v}$, где $\stackrel{e}{v} \in C^{\infty}(\omega)$ u $\stackrel{e}{v}$ әкспоненциально стремится к нулю при $\xi+\eta \rightarrow+\infty$. 
ДокаЗАТЕЛЬСтво. Найдем многочлен $R(\cdot ;(2 k, 2 k))$ такой, что $L^{+} R=0$. Затем для $\bar{v}:=v-R$ получим задачу

$$
L^{+} \bar{v}=0, \quad \bar{v}(0, \eta)=0, \quad \bar{v}(\xi, 0)=-R(\xi, 0),
$$

где $R(\xi, 0)$ - многочлен степени $2 k$.

Сделаем замену $\bar{v}=\bar{w} e^{-\eta}$. Тогда

$$
L^{-} \bar{w}=0, \quad \bar{w}(0, \eta)=0, \quad \bar{w}(\xi, 0)=-R(\xi, 0) .
$$

По лемме 2.1 найдется многочлен $S(\xi, \eta ;(2 k, 2 k))$ такой, что $\widetilde{w}:=\bar{w}-S$ удовлетворяет условиям $L^{-} \widetilde{w}=0, \widetilde{w}(\xi, 0)=0, \widetilde{w}(0, \eta)$ - многочлен степени $2 k$. Поэтому $[1 ;$ гл. IV,$(1.25)] \widetilde{w}(\cdot)=\Xi(\cdot ; k)$. Таким образом,

$$
v=R(\cdot ;(2 k, 2 k))+S(\cdot ;(2 k, 2 k)) e^{-\eta}+\Xi(\cdot ; k) e^{-\eta} .
$$

Отметим, что последнее слагаемое экспоненциально стремится к нулю при $\xi+\eta \rightarrow+\infty$.

Доказанные леммы позволяют определить вид решений рассматриваемых задач с правыми частями вида $P+Q e^{-\eta}$.

Teоpema 2.2. 1) Задача $L^{+} v(\xi, \eta)=P(\xi, \eta ;(m, k)), v(\xi, 0)=0, v(0, \eta)=0$ имеет решение вида

$$
v=R(\xi, \eta ;(m+2, m+2))+S(\xi, \eta ;(m+2, m+2)) e^{-\eta}+\stackrel{e}{v} .
$$

2) Задача $L^{+} v(\xi, \eta)=P(\xi, \eta ;(m, k)) e^{-\eta}, v(\xi, 0)=0=v(0, \eta)$ имеет решение вида $v=S(\cdot ;(m+2, k)) e^{-\eta}+\stackrel{e}{v}$.

3) Задача $L^{-} w(\xi, \eta)=P(\xi, \eta ;(m, k)) e^{-\eta}, w(\xi, 0)=0=w(0, \eta)$ имеет решение вида

$$
w=R(\xi, \eta ;(k, k))+S(\cdot ;(m, k)) e^{-\eta}+\Xi\left(\xi, \eta ; 2+2\left[\frac{k}{2}\right]\right) .
$$

Следующие классы функций призваны обеспечить решение системы (1.23) с правыми частями вида $\Xi(\cdot ; m)$, появляюшимися в силу теорем 2.1 и 2.2.

Здесь, в отличие от случая одного уравнения [7], [1], появляются логарифмические множители.

Класс $\stackrel{\circ}{\mathscr{B}}$ и его расширение $\mathscr{B}_{r, k}$ предназначены для удовлетворения граничных условий вида $\eta^{r} \ln ^{k} \eta$ при $\xi=0$.

В дальнейшем используются следуюшие обозначения:

$$
L_{0}^{-}:=\frac{\partial^{2}}{\partial \xi^{2}}-\frac{\partial}{\partial \eta}, \quad L_{0}^{+}:=\frac{\partial^{2}}{\partial \xi^{2}}+\frac{\partial}{\partial \eta} .
$$

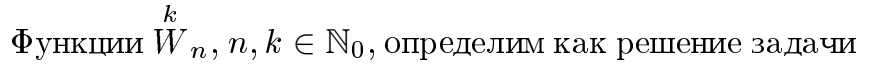

$$
L_{0}^{-\stackrel{k}{W}} \text { n }=0, \quad \stackrel{k}{W} n(0, \eta)=\eta^{n / 2} \ln ^{k} \eta, \quad \stackrel{k}{W} n(\xi, 0)=0 .
$$


Тогда [15]

$$
\stackrel{k}{W}_{n}(\xi, \eta)=\frac{1}{2 \sqrt{2}} \int_{0}^{\eta} \xi(\eta-\bar{\eta})^{-3 / 2} \exp \left(-\frac{\xi^{2}}{4(\eta-\bar{\eta})}\right) \bar{\eta}^{n / 2} \ln ^{k} \bar{\eta} d \bar{\eta} .
$$

Отсюда, сделав замену переменных $\vartheta:=\xi(2 \sqrt{(\eta-\bar{\eta})})^{-1}$, получим

$$
\begin{gathered}
\stackrel{k}{W_{n}(\xi, \eta)}=\eta^{n / 2} \sum_{s=0}^{k} \ln ^{s} \eta \Phi_{n, k, s}(\theta), \quad \theta=\frac{\xi}{2 \sqrt{\eta}}, \\
\Phi_{n, k, s}(\theta)=\alpha_{n, k, s} \int_{\theta}^{\infty} e^{-\vartheta^{2}}\left(1-\frac{\theta^{2}}{\vartheta^{2}}\right)^{n / 2} \ln ^{k-s}\left(1-\frac{\theta^{2}}{\vartheta^{2}}\right) d \vartheta:=\alpha_{n, k, s} \Psi_{n, k-s}(\theta), \\
\Psi_{n, s}(0)=0, \quad s>0, \quad \Psi_{n, s}^{\prime}(0)=0, \quad s>1 .
\end{gathered}
$$

Отметим, что $\stackrel{0}{W}_{n}(\xi, \eta)=\alpha_{n, 0,0} \eta^{n / 2} \Psi_{n, 0}(\theta)=\beta_{n} \eta^{n / 2} e^{-\theta^{2}} H_{-1-n}(\theta)$, где $H_{m}(\theta)$ - функция Эрмита [16].

Лемма 2.3. Справедливо следующее:

1) $\frac{\partial}{\partial \eta} \stackrel{k}{W}_{n}=\frac{n}{2} \stackrel{k}{W}_{n-2}+k \stackrel{k-1}{W}_{n-2}$ npu $n \geqslant 2$;

2) $\frac{\partial}{\partial \xi} \stackrel{0}{W}_{n}=C_{n} \stackrel{0}{W}_{n-1}$ npu $n \geqslant 1$;

3) $\frac{\partial}{\partial \xi} \stackrel{k}{W}_{n}=C_{n, k} \stackrel{k}{W}_{n-1}+D_{n, k} \stackrel{k-1}{W}_{n-1}$ npu $n \geqslant 1, k \geqslant 1$ u $C_{n, k} \neq 0 \neq D_{n, k}$.

ДокАЗАТЕльство следует непосредственно из определения $\stackrel{k}{W} \underset{n}{ }$.

Определим класс $\stackrel{\circ}{\mathscr{B}}$ как линейную оболочку функций $\xi^{l} \eta^{m} \stackrel{k}{W}{ }_{n}$ при $l, m$, $n, k \in \mathbb{N}_{0}$.

Лемма 2.4. Справедливо следующее:

1) $\forall f \in \stackrel{\circ}{\mathscr{B}} \exists w \in \stackrel{\circ}{\mathscr{B}}: L_{0}^{-} w=f, w(\xi, 0)=0, w(0, \eta)=0$;

2) $\forall f \in \stackrel{\circ}{\mathscr{B}} \exists v \in \stackrel{\circ}{\mathscr{B}}: L_{0}^{+} v=f$.

ДокаЗАтельство. 1) Пусть $f=\xi^{l} \eta^{m} \stackrel{k}{W} n$. Сначала при $m=0$ проводится индукция по $p=k+l$. Например, при $p=0$ решение $w$ ишется в виде $w=E \xi \stackrel{0}{W_{n+1}}$.

После этого проводится индукция по $p=l+k+m$. При этом решение $w$ ишется в виде $w=E \xi^{l+1} \eta^{m} \stackrel{k}{W} n+1+w_{1}$.

2) Это утверждение тоже доказывается индукцией по $p=l+m+k$. При этом решение $v$ ищется в виде $v=E \xi^{l} \eta^{m} \stackrel{k}{W}{ }_{n+2}+v_{1}$.

Определим класс $\mathscr{B}$ следуюшим образом $\mathscr{B}:=\left\{\partial^{p} w / \partial \eta^{p}: w \in \stackrel{\circ}{\mathscr{B}}\right\}$.

Из определения $\stackrel{k}{W}{ }_{n}$ следует, что каждая функция из $\mathscr{B}$ есть сумма функций вида $\eta^{r / 2} \sum_{s=0}^{k} \Phi_{s}(\theta) \ln ^{s} \eta$. 
Множество таких функций с фиксированными $r$ и $k$ будем обозначать через $\mathscr{B}_{r, k}$ (считаем, что $\mathscr{B}_{r, k-1} \subset \mathscr{B}_{r, k}$ ).

Через $\mathscr{E}_{n, k}$ обозначим класс функций $F \in C^{\infty}\left(\omega^{\prime}\right)$, имеющих при $\xi+\eta \rightarrow+\infty$ асимптотику вида $\sum_{s=-n}^{\infty} f_{s}, f_{s} \in \mathscr{B}_{-s, k}$, через $\mathscr{E}_{n, k, 0}-$ класс функций $\varphi \in C^{\infty}(0 ;+\infty)$ с асимптотикой $\sum_{s=-n}^{\infty} f_{s}(0 ; \eta), f_{s} \in \mathscr{B}_{-s, k}$, при $\eta \rightarrow+\infty$, а через $\mathscr{F}_{n, k}-$ класс формальных рядов $\sum_{s=-n}^{\infty} f_{s}, f_{s} \in \mathscr{B}_{-s, k}$.

Отметим, что $\xi^{l} \eta^{m} \partial^{p} \stackrel{k}{W}{ }_{n} / \partial \eta^{p} \in \mathscr{B}_{l+2 m+n-2 p, k}$ и из того, что $w \in \mathscr{B}_{r, k}$, следует, что $\partial w / \partial \xi \in \mathscr{B}_{r-1, k}, \partial^{2} w / \partial \xi^{2}, \partial w / \partial \eta \in \mathscr{B}_{r-2, k}, \partial^{2} w / \partial \eta^{2} \in \mathscr{B}_{r-4, k}$.

Из леммы 2.3 и определения $\mathscr{B}_{r, k}$ вытекает

Tеорема 2.3. Справедливо следующее:

1) $\forall f \in \mathscr{B}_{r, k} \exists w \in \mathscr{B}_{r+2, k}: L_{0}^{-} w=f, w(\xi, 0)=0, w(0, \eta)=0$;

2) $\forall f \in \mathscr{B}_{r, k} \exists v \in \mathscr{B}_{r+2, k}: L_{0}^{+} v=f$;

3) $\forall F \in \mathscr{E}_{n-2, k}, \forall \varphi \in \mathscr{E}_{n, k, 0} \exists w \in \mathscr{F}_{n, k}$ ф.a.p. задачи $L^{-} w=F, w(\xi, 0)=0$, $w(0, \eta)=\varphi(\eta)$

4) $\forall F \in \mathscr{E}_{n-2, k} \quad \exists v \in \mathscr{F}_{n, k}$ ф.а.p. уравнения $L^{+} v=F$.

Решение уравнения $L^{+} v=f \in \mathscr{B}_{r, k}$ из класса $\mathscr{B}_{r+2, k}$ при $\xi=0$ имеет вид $\eta^{r / 2} \ln ^{k} \eta$. Функции классов $\stackrel{\circ}{\mathscr{B}}^{+}$и $\mathscr{B}_{r, k}^{+}$призваны убрать невязку такого вида на гранище $\xi=0$.

Рассмотрим задачу $L_{0}^{+} V=0, V(0, \eta)=\eta^{n / 2}, V$ - функция медленного роста.

Ищем $V$ в виде $V=\eta^{n / 2} G(\theta)$, где $\theta:=\xi /(2 \sqrt{\eta})$.

Тогда для $G$ получим уравнение $G^{\prime \prime}-2 \theta G^{\prime}+2 n G=0, G(0) \neq 0$. Поэтому $G=H_{n}(\theta)$ - функция Эрмита порядка $n$ [16]. При $n \in \mathbb{N}_{0}$ это многочлен степени $n$, четный при четных $n$ и нечетный при нечетных $n$. При этом $H_{n}(0)=\Gamma(-n / 2) /(2 \Gamma(-n))$. Тем самым $H_{2 k+1}(0)=0$ и решения рассматриваемой задачи вида $\eta^{n / 2} G(\theta)$ не существует.

При $n=2 k, k \in \mathbb{N}_{0}$, имеем $V=M \eta^{k} H_{2 k}(\xi /(2 \sqrt{\eta}))$ - многочлен от $\xi$ и $\eta$, параболически однородный степени $2 k$. При $n \in-\mathbb{N}$ получим $V=M_{n}^{\prime} \eta^{n / 2} H_{n}(\theta)=$ $M_{n} \eta^{n / 2}\left(H_{-1}(\theta)\right)^{(1-n)}$ и $H_{n}(\theta)=\sum_{s=0}^{\infty} h_{2 s} \theta^{n-2 s}$ при $\theta \rightarrow+\infty$.

Найдем функцию $\stackrel{k}{V}$ вида

$$
\stackrel{k}{V}{ }_{1}=\eta^{1 / 2}\left(G_{0}(\theta) \ln ^{k} \eta+G_{1}(\theta) \ln ^{k-1} \eta+\cdots+G_{k}(\theta)\right),
$$

удовлетворяющую уравнению $L_{0}^{+} \stackrel{k}{V}{ }_{1}=0$.

Тогда функции $G_{s}$ - решения следуюшей системы л.о.д.у.

$$
L G_{0}=0, L G_{1}+k G_{0}=0, L G_{2}+(k-1) G_{1}=0, \ldots, L G_{k}+G_{k-1}=0,
$$

где

$$
L:=\frac{1}{4} \frac{d^{2}}{d \theta^{2}}-\frac{1}{2} \theta \frac{d}{d \theta}+\frac{1}{2} .
$$

Рассмотрим медленно растущие функции, определенные аналогично (2.1), $L g_{0}=0, L g_{1}=-g_{0}, L g_{2}=-g_{1}, \ldots, L g_{k}=-g_{k-1}$. 
Тогда $g_{0}=\theta$. После замены $g_{s}=\theta \varphi_{s}$ для $\varphi_{s}$ получим уравнение

$$
\varphi_{s}^{\prime \prime}-2\left(\theta-\frac{1}{\theta}\right) \varphi_{s}^{\prime}=-\frac{4}{\theta} g_{s-1}
$$

откуда

$$
\varphi_{s}^{\prime}=4 \theta^{-2} e^{\theta^{2}} \int_{\theta}^{\infty} \vartheta e^{-\vartheta^{2}} g_{s-1}(\vartheta) d \vartheta .
$$

Из (2.2) при $s=1$ получим

$$
\varphi_{1}^{\prime}=4 \theta^{-2} e^{\theta^{2}} \int_{\theta}^{\infty} \vartheta^{2} e^{-\vartheta^{2}} d \vartheta
$$

Тогда $\varphi_{1}^{\prime}=4 C_{1} \theta^{-2}+O(1)$ при $\theta \rightarrow 0$, где

$$
C_{1}=\int_{\theta}^{\infty} \vartheta^{2} e^{-\vartheta^{2}} d \vartheta=\frac{\sqrt{\pi}}{4} .
$$

Выписывая асимптотику интеграла из $(2.3)$ при $\theta \rightarrow+\infty$, получаем $\varphi_{1}^{\prime}=2 \theta^{-1}+$ $\mathscr{R}_{-3}(\theta)$ при $\theta \rightarrow+\infty$, где через $\mathscr{R}_{k}$ обозначены функции с асимптотикой $\mathscr{R}_{k}(\theta)=\sum_{s=0}^{\infty} \beta_{s} \theta^{k-2 s}$ при $\theta \rightarrow+\infty$.

Возьмем окончательно

$$
\varphi_{1}=2 \ln \theta-\int_{\theta}^{\infty}\left(\varphi_{1}^{\prime}(\vartheta)-2 \vartheta^{-1}\right) d \vartheta=: 2 \ln \theta+2 F_{1}(\theta) .
$$

Поэтому $g_{1}=2 \theta \ln \theta+2 \theta F_{1}(\theta), g_{1}=-\sqrt{\pi}+O(\theta)$ при $\theta \rightarrow 0$ и $F_{1}(\theta)=\mathscr{R}_{-2}(\theta)$ при $\theta \rightarrow+\infty$.

Продолжая этот процесс, получаем $g_{s}=\left(2^{s} / s !\right) \theta \ln ^{s} \theta+2 \theta F_{s}(\theta), g_{s}=$ $-4 C_{s}+O(\theta)$ при $\theta \rightarrow 0, C_{s}=\int_{0}^{\infty} \vartheta e^{-\vartheta^{2}} g_{s-1}(\vartheta) d \vartheta$ и $F_{s}(\theta)=\ln ^{s-1} \theta \mathscr{R}_{-2}(\theta)+$ $\ln ^{s-2} \theta \mathscr{R}_{-2}(\theta)+\cdots+\mathscr{R}_{-2}(\theta)$ при $\theta \rightarrow+\infty$.

Теперь, положив $G_{0}:=g_{0}$, получаем, что $G_{s}=A_{k}^{s} g_{s}$, где $A_{k}^{s}-$ число всех размешений из $k$ по $s$.

Таким образом, окончательно

$$
\stackrel{k}{V}_{1}=\eta^{1 / 2} \sum_{s=0}^{k} A_{k}^{s}\left(\frac{2^{s}}{s !} \theta \ln ^{s} \theta+2 \theta F_{s}(\theta)\right) \ln ^{k-s} \eta,
$$

где $F_{0}(\theta) \equiv 0$.

Переписьвая формулу (2.4) в переменных $\xi$ и $\theta$, получаем

$$
\stackrel{k}{V_{1}}=2^{k-1} \xi \ln ^{k} \frac{\xi}{2}+\sum_{s=1}^{k} \bar{F}_{s}(\theta) \ln ^{s} \xi,
$$

где $\bar{F}_{s}(\theta)=\sum_{\sigma=0}^{k-s-1} \mathscr{R}_{-2, \sigma}(\theta) \ln ^{\sigma} \theta$.

Определим $\stackrel{k}{V}$ следуюшим образом:

$$
\begin{gathered}
\stackrel{k}{V}{ }_{2 m+1}(\xi, \eta):=\int_{0}^{\eta} \stackrel{k}{V}_{2 m-1}(\xi, \tau) d \tau-\int_{0}^{\xi} d \xi_{1} \int_{0}^{\xi_{1}} \stackrel{k}{V}_{2 m-1}\left(\xi_{2}, 0\right) d \xi_{2}, \\
\stackrel{k}{V}{ }_{2 m}:=\frac{\partial}{\partial \xi} \stackrel{k}{V}{ }_{2 m+1} .
\end{gathered}
$$

Непосредственно из (2.4)-(2.6) следует 
ЛЕмма 2.5. Справедливо следующее:

1) $L_{0}^{+} \stackrel{k}{V}_{n}=0, \stackrel{k}{V}_{n}(0, \eta)=\eta^{n / 2} P(\ln \eta ; k), \stackrel{k}{V}_{n}(\xi, 0)=\xi^{n} Q(\ln \xi ; k) ;$

2)

$$
\begin{aligned}
V_{2 m}^{k} & =\eta^{m} P\left(\theta^{2} ; m\right) \ln ^{k} \eta+\eta^{m} \sum_{s=0}^{k-1} F_{2 m, k, s}(\theta) \ln ^{s} \eta \\
& =\sum_{s=0}^{k} Q_{s}(\xi, \eta ;(2 m, o)) \ln ^{s} \xi+\xi^{2 m} \sum_{s=0}^{k-1} \bar{F}_{2 m, k, s}(\theta) \ln ^{s} \xi, \\
F_{2 m, k, s}(\theta) & =\sum_{\sigma=s}^{k} Q\left(\theta^{2} ; m\right) \ln ^{\sigma-s} \theta+\sum_{\sigma=0}^{k-s-1} \mathscr{R}{ }_{-2}(\theta) \ln ^{\sigma} \theta \quad n p u \quad \theta \rightarrow+\infty, \\
\bar{F}_{2 m, k, s}(\theta) & =\sum_{\sigma=0}^{k-s-1} \mathscr{R} \mathscr{L}_{-2 m}(\theta) \ln ^{\sigma} \theta \quad n p u \quad \theta \rightarrow+\infty .
\end{aligned}
$$

Пусть опять $\stackrel{\circ}{\mathscr{B}}+$ линейная оболочка функций вида $\xi^{l} \eta^{m} \stackrel{k}{V}_{n}$ при $l, m, n, k \in \mathbb{N}_{0}$, a $\mathscr{B}^{+}:=\left\{\partial^{p} v / \partial \eta^{p}: v \in \stackrel{\circ}{\mathscr{B}}^{+}\right\}$.

И в этом случае если $v \in \mathscr{B}^{+}$, то $v$ - сумма функций вида $\eta^{r / 2} \sum_{s=0}^{k} Y_{s}(\theta) \ln ^{s} \eta$. Поэтому снова класс таких функций обозначим через $\mathscr{B}_{r, k}^{+}$.

Аналогично предыдущему определяются классы $\mathscr{E}_{n, k}^{+}, \mathscr{E}_{n, k, 0}^{+}$и $\mathscr{F}_{n, k}^{+}$.

Поскольку непосредственно из определения $\partial \stackrel{k}{V}{ }_{n} / \partial \xi=\stackrel{k}{V}_{n-1}, \partial \stackrel{k}{\partial}{ }_{n} / \partial \eta=\stackrel{k}{V}_{n-2}$, то справедливы аналоги леммы 2.4 и теоремы 2.3 .

Теорема 2.4. Справедливо следующее:

1) $\forall f \in \mathscr{B}_{r, k}^{+} \exists v \in \mathscr{B}_{r+2, k}^{+}: L_{0}^{+} v=f, v(0, \eta)=0$ npu $\eta>0$;

2) $\forall f \in \mathscr{B}_{r, k}^{+} \exists w \in \mathscr{B}_{r+2, k}^{+}: L_{0}^{-} w=f$;

3) $\forall F \in \mathscr{E}_{n-2, k}^{+}, \forall \varphi \in \mathscr{E}_{n, k, 0}^{+} \exists v \in \mathscr{F}_{n, k}^{+}$ф.a.p. задачu $L^{+} v=F, w(\xi, 0)=0$, $v(0, \eta)=\varphi(\eta)$

4) $\forall F \in \mathscr{E}_{n-2, k}^{+} \exists w \in \mathscr{F}_{n, k}^{+}$ф.а.р. уравнения $L^{-} w=F$.

Функции классов $\stackrel{\circ}{\mathscr{B}}^{+}$и $\mathscr{\mathscr { B }}_{r, k}^{+}$ликвидируют невязку на границе $\xi=0$, но привносят свою степенную невязку при $\eta=0$. Класс $\stackrel{\circ}{\mathscr{B}}^{*}$ и его расширение $\mathscr{B}^{*}$ призваны ликвидировать эту новую невязку.

Определим $\stackrel{k}{W}{ }_{2 m+1}^{*}$ как решение задачи

$$
\begin{gathered}
L_{0}^{-} W=0, \quad V(0, \eta)=0, \quad W(\xi, 0)=\xi^{2 m+1} \ln ^{k} \xi, \quad \xi>0, \\
W(-\xi, 0)=-W(\xi, 0) .
\end{gathered}
$$

Тогда $[18]$

$$
\stackrel{k}{W_{2 m+1}^{*}}(\xi, \eta)=\frac{1}{2 \sqrt{\pi \eta}} \int_{0}^{\infty}\left(\exp \left(-\frac{(\xi-\bar{\xi})^{2}}{4 \eta}\right)-\exp \left(-\frac{(\xi+\bar{\xi})^{2}}{4 \eta}\right)\right) \bar{\xi}^{2 m+1} \ln ^{k} \bar{\xi} d \bar{\xi} .
$$


Положим $\stackrel{k}{W} \underset{2 m}{*}:=\partial \stackrel{k}{W_{2 m+1}^{*}} / \partial \xi$.

Так как при $2 m+1>1$ функция $W:=\partial \stackrel{k}{W_{2 m+1}^{*}} / \partial \eta$ удовлетворяет задаче

$$
\begin{gathered}
L_{0}^{-} W=0, \quad W(0, \eta)=\frac{\partial}{\partial \eta} \stackrel{k}{W}{ }_{2 m+1}^{*}(0, \eta)=0, \\
W(\xi, 0)=\frac{\partial^{2} \stackrel{k}{\partial \xi^{2}} W_{2 m+1}^{*}(\xi, 0)}{=\xi^{n-2}\left(n(n-1) \ln ^{k} \xi+(2 n k-k) \ln ^{k-1} \xi+k(k-1) \ln ^{k-2} \xi\right),}
\end{gathered}
$$

$n=2 m+1$, то отсюда вытекает

Лемма 2.6. Справедливо следующее:

1) $\frac{\partial}{\partial \eta} \stackrel{k}{W}{ }_{2 m+1}^{*}=2 m(2 m+1) \stackrel{k}{W_{2 m-1}^{*}}+k(4 m+1) \stackrel{k-1}{W_{2 m-1}^{*}}+k(k-1) \stackrel{k-2}{W} \underset{2 m-1}{*}$;

2) $\frac{\partial}{\partial \xi} \stackrel{k}{W}_{2 m+1}^{*}=C_{n, k, 0} \stackrel{k}{W}{ }_{n-1}^{*}+C_{n, k, 1} \stackrel{k-1}{W} \underset{n-1}{*}+C_{n, k, 2} \stackrel{k-2}{W} \underset{n-1}{*} u C_{n, k, 0} \neq 0$.

Классы $\stackrel{\circ}{\mathscr{B}}{ }^{*}$ и $\mathscr{B}^{*}$ определяюся аналогично классам $\stackrel{\circ}{\mathscr{B}}$ и $\mathscr{\mathscr { B }}$.

Переходя в (2.7) к переменньм $\eta$ и $\theta$, получаем

$$
\stackrel{k}{W}_{n}^{*}=\eta^{n / 2} \sum_{s=0}^{k} \beta_{n, k, s}\left(Y_{n, k-s}(\theta)-\Psi_{n, k-s}(\theta)\right) \ln ^{s} \eta,
$$

где $Y_{2 m, l}(\theta):=Y_{2 m+1, l}^{\prime}(\theta), \Psi_{2 m, l}(\theta):=\Psi_{2 m+1, l}^{\prime}(\theta)$,

$$
\begin{aligned}
& Y_{2 m+1, l}(\theta)=\int_{-\theta}^{\infty} e^{-\vartheta^{2}}(\vartheta+\theta)^{2 m+1} \ln ^{l}(2(\vartheta+\theta)) d \vartheta, \\
& \Psi_{2 m+1, l}(\theta)=\int_{\theta}^{\infty} e^{-\vartheta^{2}}(\vartheta-\theta)^{2 m+1} \ln ^{l}(2(\vartheta-\theta)) d \vartheta .
\end{aligned}
$$

Аналогично, переходя в $(2.7)$ к переменным $\xi$ и $\theta$, получаем

$$
\stackrel{k}{W}_{n}^{*}=\xi^{n} \sum_{s=0}^{k} \gamma_{n, k, s}\left(X_{n, k-s}(\theta)-\Upsilon_{n, k-s}(\theta)\right) \ln ^{s} \xi,
$$

где

$$
\begin{aligned}
X_{2 m, l}(\theta):= & (2 m+1) X_{2 m+1, l}(\theta)+(k-l+1) X_{2 m+1, l-1}(\theta)+\theta X_{2 m+1, l}^{\prime}(\theta), \\
\Upsilon_{2 m, l}(\theta):= & (2 m+1) \Upsilon_{2 m+1, l}(\theta)+(k-l+1) \Upsilon_{2 m+1, l-1}(\theta)+\theta \Upsilon_{2 m+1, l}^{\prime}(\theta), \\
& X_{2 m+1, l}(\theta)=\int_{-\theta}^{\infty} e^{-\vartheta^{2}}\left(1+\frac{\vartheta}{\theta}\right)^{2 m+1} \ln ^{l}\left(1+\frac{\vartheta}{\theta}\right) d \vartheta, \\
& \Upsilon_{2 m+1, l}(\theta)=\int_{\theta}^{\infty} e^{-\vartheta^{2}}\left(\frac{\vartheta}{\theta}-1\right)^{2 m+1} \ln ^{l}\left(\frac{\vartheta}{\theta}-1\right) d \vartheta .
\end{aligned}
$$

Отметим также, что в силу $(2.7) \stackrel{k}{W_{2 m}^{*}}(0, \eta)=\eta^{m} P(\ln \eta ; k)$.

Из (2.8) имеем: если $w \in \mathscr{B}^{*}$, то $w$ - сумма функций вида $\eta^{r / 2} \sum_{s=0}^{k} \widetilde{Y}_{s}(\theta) \ln ^{s} \eta$. Множество функций из $\mathscr{B}^{*}$ такого вида обозначим через $\mathscr{B}_{r, k}^{*}$.

Соответственно определяются и классы $\mathscr{E}_{n, k}^{*}, \mathscr{E}_{n, k, 0}^{*}$ и $\mathscr{F}_{n, k}^{*}$.

Верны аналоги леммы 2.4 и теоремы 2.3. 
Теорема 2.5. Справедливо следующее:

1) $\forall f \in \mathscr{B}_{r, k}^{*} \exists w \in \mathscr{B}_{r+2, k}^{*}: L_{0}^{-} w=f, w(0, \eta)=0$ npu $\eta>0$ (uлu $w(\xi, 0)=0$ npu $\xi>0)$;

2) $\forall f \in \mathscr{B}_{r, k}^{*} \exists v \in \mathscr{B}_{r+2, k}^{*}: L_{0}^{+} v=f$;

3) $\forall F \in \mathscr{E}_{n-2, k}^{*}, \forall \varphi \in \mathscr{E}_{n, k, 0}^{*} \exists w \in \mathscr{F}_{n, k}^{*}$ ф.а.p. задачи $L^{-} w=F, w(0, \eta)=$ $\varphi(\eta)$

4) $\forall F \in \mathscr{E}_{n-2, k}^{*} \exists v \in \mathscr{F}_{n, k}^{*}$ ф.а.р. уравнения $L^{+} v=F$;

5) $\forall F \in \mathscr{E}_{n, k}^{*}, \forall \varphi \in \mathscr{E}_{n, k, 0}^{*} \exists w \in e^{-\eta \mathscr{F}_{n, k}^{*}}$ ф.a.p. задачu $L^{-} w=e^{-\eta} F$, $w(0, \eta)=\varphi(\eta)$

6) $\forall F \in \mathscr{E}_{n-2, k}^{*}, \forall \varphi \in \mathscr{E}_{n, k, 0}^{*} \exists v \in e^{-\eta \mathscr{F}_{n, k}^{*}}$ ф.a.p. задачu $L^{+} v=e^{-\eta} F$.

Непосредственно из определения следует $Y_{n, 0}(\theta)=\Phi(\theta)+\theta^{n} P\left(\theta^{-2} ;[n / 2]\right)$, $Y_{n, l}(\theta)=\mathscr{Q}_{n, l}(\theta), X_{n, 0}(\theta)=\Phi(\theta)+\theta^{-2[n / 2]} P\left(\theta^{2} ;[n / 2]\right), X_{n, l}(\theta)=\mathscr{Q}_{-2[(l+1) / 2], 0}(\theta)$.

Здесь через $\mathscr{Q}_{n, l}(\theta)$ обозначены гладкие функции, разлагающиеся при $\theta \rightarrow+\infty$ в асимптотические ряды вида $\sum_{s=0}^{\infty} \theta^{n-s-2} P_{s}(\ln \theta ; l)$.

Это позволяет найти асимптотику при $\theta \rightarrow+\infty$ функций из $\mathscr{B}_{r, k}^{*}$.

Tеорема 2.6. Ecлu $w \in \mathscr{B}_{r, k}^{*}$, mo $w=\sum_{s=0}^{\infty} \xi^{r-2 s} \eta^{s} P_{s}(\ln \xi ; k) n p u \eta \leqslant$ $\xi \rightarrow+\infty$, и если $r<0$, mо $P_{s}(\cdot ; k)=P_{s}(\cdot ; k-1)$.

Может случиться, что некоторые $P_{s}(\cdot ; k) \equiv 0$ и тогда главный член асимптотики функции $w \in \mathscr{B}_{r, k}^{*}$ будет иметь вид $\xi^{\alpha} \eta^{\beta} P(\ln \xi ; k), \alpha+2 \beta=r$. В этом случае положим $\operatorname{Ord}_{\xi}(w):=\alpha$.

ЛЕмма 2.7. Справедливо следующее: $\forall f \in \mathscr{B}_{r, k}^{*} \quad \exists w \in \mathscr{B}_{r+2, k}^{*}: L_{0}^{-} w=f u$ $\operatorname{Ord}_{\xi}(w) \leqslant \operatorname{Ord}_{\xi}(f)$.

ДокАЗАТЕЛЬСтво. Прежде всего отметим, что в силу теоремы 2.6

$$
\operatorname{Ord}_{\xi}\left(\xi^{l} \eta^{m} \frac{\partial^{p}}{\partial \eta^{p}} \stackrel{k}{W} \underset{n}{*}\right)=l+n-2 p
$$

Пусть $f=\xi^{l} \eta^{m} \partial^{p} \stackrel{k}{W_{n}^{*}} / \partial \eta^{p}$. Проведем индукцию по $l$. Пусть $l=0$. Тогда $w=K \eta^{m+1} \partial^{p} \stackrel{k}{W}_{n}^{*} / \partial \eta^{p}$ при некотором $K$ и $\operatorname{Ord}_{\xi}(w) \leqslant \operatorname{Ord}_{\xi}(f)$.

Пусть теперь $l>0, n>0$. Ищем $w$ в виде $w=K \xi^{l} \eta^{m+1} \partial^{p} \stackrel{k}{W_{n}^{*}} / \partial \eta^{p}+w_{1}$. Тогда для $w_{1}$ при соответствуюшем выборе $K$ получим задачу

$L_{0}^{-} w_{1}=c_{1} \xi^{l-2} \eta^{m+1} \frac{\partial^{p}}{\partial \eta^{p}} \stackrel{k}{W_{n}^{*}}+\xi^{l-1} \eta^{m+1} \frac{\partial^{p}}{\partial \eta^{p}}\left(c_{2} \stackrel{k}{W}_{n-1}^{*}+c_{3} \stackrel{k-1}{W}_{n-1}^{*}+c_{4} \stackrel{k-2}{W}_{n-1}^{*}\right)$.

По предположению индукции $\operatorname{Ord}_{\xi}\left(w_{1}\right) \leqslant l-2+n-2 p$.

Если $n=0$, то последнее слагаемое имеет вид

$$
c_{2} \xi^{l-1} \eta^{m+1} \frac{\partial^{p+1}}{\partial \eta^{p} \partial \xi} \stackrel{k}{W} \underset{0}{*}=c_{2} \xi^{l-1} \eta^{m+1} \frac{\partial^{p+2}}{\partial \eta^{p} \partial \xi^{2}} \stackrel{k}{W}{ }_{1}^{*}=c_{2} \xi^{l-1} \eta^{m+1} \frac{\partial^{p+1}}{\partial \eta^{p+1}} \stackrel{k}{W}{ }_{1}^{*}
$$

и предположение индукции применимо.

Теперь покажем, что формальным асимптотическим рядам для задач $L^{-} w=f$, $\left.w\right|_{\partial \omega}=0$ и $L^{+} v=g,\left.v\right|_{\partial \omega}=0$ соответствуют истинные решения этих задач с аналогичной асимптотикой при $\xi+\eta \rightarrow+\infty$. 
ЛЕмма 2.8. Если $f \in \mathscr{B}_{r, k}, w=\eta^{1+r / 2} \sum_{s=0}^{k} \Phi_{s}(\theta) \ln ^{s} \eta u L_{0}^{-} w=f$ в $\omega^{\prime}$, mo $w \in \mathscr{B}_{r+2, k}$.

ДоКАЗАТЕЛЬСТВо аналогично [1; гл. IV, лемма 1.3]. В силу леммы 2.4 найдется $w_{1} \in \mathscr{B}_{r+2, k}$ такое, что $L_{0}^{-} w_{1}=f,\left.w_{1}\right|_{\partial \omega}=0$. Тогда $w_{2}:=w-w_{1}$ удовлетворяет однородному уравнению $L_{0}^{-} w_{2}=0$ и $w_{2}(0, \eta)=\eta^{1+r / 2} P(\ln \eta ; k)$, т.е. $w_{2} \in \mathscr{B}_{r+2, k}$.

Tеорема 2.7. Пусть $q \in C^{\infty}(\omega) u \sum_{s=1}^{\infty} v_{s}-$ ф.а.p. задачи $L^{+} v=q,\left.v\right|_{\partial \omega}=0$ npu $\xi+\eta \rightarrow+\infty$, m.e. $L^{+} \sum_{s=1}^{N} v_{s}-q=O\left((\xi+\eta)^{-N_{1}}\right),\left.\sum_{s=1}^{N} v_{s}\right|_{\partial \omega}=$ $O\left((\xi+\eta)^{-N_{1}}\right)$ и, наконеи, $v_{s} \in C^{\infty}\left(\omega^{\prime}\right), v_{N}=O\left((\xi+\eta)^{-N_{1}}\right)$ при $\xi+\eta \rightarrow+\infty$, где $N_{1} \rightarrow+\infty$ при $N \rightarrow+\infty$.

Тогда существует $v$ - решение указанной задачи, для которого $\sum_{s=1}^{\infty} v_{s}$ есть его асимптотическое разложсение при $\xi+\eta \rightarrow+\infty$.

ДокаЗАтЕльство. Отметим, что $v_{s}$ могут иметь особенности в точке $(0 ; 0)$.

Пусть $\widehat{v}_{N}:=\sigma(\xi, \eta) \sum_{s=1}^{N} v_{s}$, где $\sigma$ - “срезаюшая" функция, равная нулю в некоторой окрестности точки $(0 ; 0)$ и единище вне некоторого компакта. Тогда $\widehat{F}_{N}:=L^{+} \widehat{v}_{N}$ не имеет особенностей в точке $(0 ; 0)$ и $F_{N}:=F-\widehat{F}_{N}=O\left((\xi+\eta)^{-N_{1}}\right)$ при $\xi+\eta \rightarrow+\infty$.

Пусть $v_{N}$ - решение задачи $L^{+} v_{N}=F_{N},\left.v_{N}\right|_{\partial \omega}=-\left.\widehat{v}_{N}\right|_{\partial \omega}$, найденное по формулам (1.26)-(1.28). Тогда в силу лемм 1.2 и 1.4 справедливо соотношение $v_{N}=O\left((\xi+\eta)^{-N_{1}+2}\right)$. Так как $V_{N, p}:=\left(v_{N}+\widehat{v}_{N}\right)-\left(v_{N+p}+\widehat{v}_{N+p}\right)$ удовлетворяет условиям $L^{+} V_{N, p}=0,\left.V_{N, p}\right|_{\partial \omega}=0$ и $V_{N, p}=O\left((\xi+\eta)^{-N_{1}+2}\right)$, то $V_{N, p}$ ограничено и, поэтому, $V_{N, p}=0$, т.е. $v_{N}+\widehat{v}_{N}$ не зависит от $N$ и является искомым решением.

Teорема 2.8. Пусть $F \in C^{\infty}(\omega)$ и имеет при $\xi+\eta \rightarrow+\infty$ асимптотическое разложение вида

$$
\begin{aligned}
& F=\sum_{s=\alpha}^{\infty} f_{s}+\sum_{s=\alpha^{+}}^{\infty} f_{s}^{+}+\sum_{s=\alpha^{*}}^{\infty} f_{s}^{*}+e^{-\eta} \sum_{s=\beta}^{\infty} g_{s}^{*}, \\
& f_{s} \in \mathscr{B}_{-s, k}, \quad f_{s}^{+} \in \mathscr{B}_{-s, k}^{+}, \quad f_{s}^{*}, g_{s}^{*} \in \mathscr{B}_{-s, k}^{*},
\end{aligned}
$$

a

$$
\begin{gathered}
\sum_{s=\alpha+2}^{\infty} w_{s}+\sum_{s=\alpha^{+}+2}^{\infty} w_{s}^{+}+\sum_{s=\alpha^{*}+2}^{\infty} w_{s}^{*}+e^{-\eta} \sum_{s=\beta}^{\infty} \widetilde{w}_{s}^{*}, \\
w_{s} \in \mathscr{B}_{-s, k}, \quad w_{s}^{+} \in \mathscr{B}_{-s, k}^{+}, \quad w_{s}^{*}, \widetilde{w}_{s}^{*} \in \mathscr{B}_{-s, k}^{*},
\end{gathered}
$$

- ф.а.р. задачи $L^{+} w=F,\left.w\right|_{\partial \omega}=0$ при $\xi+\eta \rightarrow+\infty$, в следующем смисле:

$$
\begin{gathered}
L^{-}\left(\sum_{s=\alpha}^{N} w_{s}^{(+, *)+2}\right)-\sum_{s=\alpha}^{N} f_{s}^{(+, *)}=O\left((\xi+\eta)^{-N_{1}}\right), \\
L^{-}\left(\sum_{s=\beta+2}^{N} \widetilde{w}_{s}^{*}\right)-\sum_{s=\beta}^{N} g_{s}^{*}=O\left((\xi+\eta)^{-N_{1}}\right), \\
\left.\left(\sum_{s=\alpha+2}^{\infty} w_{s}+\sum_{s=\alpha^{+}+2}^{\infty} w_{s}^{+}+\sum_{s=\alpha^{*}+2}^{\infty} w_{s}^{*}+e^{-\eta} \sum_{s=\beta}^{\infty} \widetilde{w}_{s}^{*}\right)\right|_{\partial \omega}=O\left((\xi+\eta)^{-N_{1}}\right) .
\end{gathered}
$$


Тогда существует $w$ - решение указанной задачи, имеющее при $\xi+\eta \rightarrow+\infty$ следующее асимптотическое разложение:

$$
w=\sum_{s=\alpha+2}^{\infty} w_{s}+\sum_{s=\alpha^{+}+2}^{\infty} w_{s}^{+}+\sum_{s=\alpha^{*}+2}^{\infty} w_{s}^{*}+e^{-\eta} \sum_{s=\beta}^{\infty} \widetilde{w}_{s}^{*}+\sum_{s=0}^{\infty} \bar{w}_{s}, \quad \bar{w}_{s} \in \mathscr{B}_{-s, k} .
$$

ДокАЗАТЕЛЬство этой теоремы аналогично доказательству теоремы 2.7 с заменой лемм 1.2 и 1.4 на лемму 1.4 из [1; гл. IV] и применением леммы 2.8 для доказательства принадлежности функций вида $\eta^{r / 2} \Phi(\theta)$ классу $\mathscr{B}_{r, 0}$.

Общий вид решений системы (1.23) со степенньми особенностями на бесконечности дает следующая

Теорема 2.9. У системы (1.23) при фиксированном наборе $\left\{\lambda_{k, l}\right\}$ существуют решения $\left\{w_{k, l}^{-}\right\}$u $\left\{v_{k, l}^{-}\right\}$со следующей асимптотикой при $\xi+\eta \rightarrow+\infty$.

1) $E$ слли $k=4 m+2 n+\delta, m \in \mathbb{N}_{0}, n, \delta \in\{0,1\}, m o$

$$
w_{k, 0}^{-}=\stackrel{\circ}{\Xi}(k, 2,5,2,4,3), \quad v_{k, 0}^{-}=\stackrel{\circ}{\Xi}(k, 0,2,0,1,1),
$$

$2 \partial e$

$$
\begin{aligned}
\stackrel{\circ}{\Xi}(k, a, b, c, d, e)=R( & ;(k, k-a))+e^{-\eta} S(\cdot ;(\bar{k}-b+3 \delta, k-c))+\Xi(\cdot ; \widetilde{k}, 0) \\
& +\sum_{s=1}^{m}\left(\Xi(\cdot ; \widetilde{k}-2-2 s, s)+\Xi^{+}(\cdot ; \widetilde{k}-1-2 s, s)\right. \\
& \left.+\Xi^{*}(\cdot ; \widetilde{k}-1-2 s, s)+e^{-\eta} \Xi^{*}(\cdot ;(\bar{k}-d-4 s, \widetilde{k}-e-2 s), s)\right), \\
\bar{k}=6 m+ & 3 n, \quad \widetilde{k}=4 m+2 n, \quad \Xi^{(+, *)}(\cdot ; k, s) \in \mathscr{E}_{k, s}^{(+, *)},
\end{aligned}
$$

а через $\Xi^{*}(\cdot ;(k, p), s)$ обозначень функиии класса $C^{\infty}(\omega)$ с асимптотикой при $\xi+\eta \rightarrow+\infty$ вида $\Xi^{*}(\cdot ;(k, p), s)=\sum_{\sigma=-k}^{\infty} f_{\sigma}, f_{\sigma} \in \mathscr{B}_{-\sigma, s}^{*}, \operatorname{Ord}_{\xi}\left(f_{\sigma}\right) \leqslant p$.

2) Функции $w_{k, l}^{-}$имеют вид, аналогичный функциям $w_{k-2-l, 0}^{-}$, a $v_{k, l}^{-}$аналогичны $v_{k-2-l, 0}^{-}$.

При этом функции $w_{2 k+1+\delta, 0}^{-}$u $v_{2 k+1+\delta, 0}^{-}$определень с точностью до слагаемых вида $R(\cdot ;(2 k+1,2 k+1))+e^{-\eta} S(\cdot ;(2 k+1,2 k+1))$, однозначно определяемых многочленом степени $k$ от переменной $\eta$.

ДоКАЗАТЕЛЬСТВо проводится индукцией по $m$. При этом можно "распараллелить" процесс доказательства и рассмотреть слагаемые вида $R+e^{-\eta} S$ и все остальные. В силу свойств классов $\mathscr{B}_{r, k}^{(+, *)}$ вид решений будут определять следующие слагаемые из правой части системы (1.23): $w_{k-2,0}^{-}, v_{k-2,0}^{-}, \xi \partial w_{k-2,0}^{-} / \partial \eta$ и $\xi \partial v_{k-2,0}^{-} / \partial \eta$

Учитьвая асимптотику функций из классов $\mathscr{B}^{(+, *)}$ (см. лемму 2.5 и теорему 2.6), получаем вид асимптотики функций $\Xi^{(+, *)}(\cdot ; n, k)$ и $\Xi^{*}(\cdot ;(n, p), k)$. 
ТЕОРема 2.10. Справедливы следующие асимптотические разложения при $\xi+\eta \rightarrow+\infty, \theta:=\xi /(2 \sqrt{\eta}) \rightarrow+\infty:$

$$
\begin{aligned}
\Xi(\xi, \eta ; n, k) & =\sum_{s=0}^{k} \ln ^{s} \eta \sum_{\sigma=0}^{\infty} \eta^{(n-\sigma) / 2} \Phi_{k, s, \sigma}(\theta), \\
\Xi^{+}(\xi, \eta ; n, k) & =\sum_{s=0}^{k} \ln ^{s} \eta \sum_{\sigma=0}^{\infty} \eta^{(n-\sigma) / 2} \mathscr{Q}_{k, s, \sigma}(\theta ; n-\sigma, k-s) \\
& =\sum_{s=0}^{k} \ln ^{s} \xi \sum_{\sigma=0}^{\infty} \xi^{n-\sigma} \mathscr{Q}_{k, s, \sigma}(\theta ; 0, k-s-1), \\
\Xi^{*}(\xi, \eta ; n, k)= & \sum_{s=0}^{k} \ln ^{s} \eta \sum_{\sigma=0}^{\infty} \eta^{(n-\sigma) / 2} \mathscr{Q}_{k, s, \sigma}(\theta ; n-\sigma, k-s) \\
= & \sum_{s=0}^{k} \ln ^{s} \xi \sum_{\sigma=0}^{\infty} \xi^{n-\sigma} \mathscr{Q}_{k, s, \sigma}(\theta ; 0,0), \\
\Xi^{*}(\xi, \eta,(n, p), k)= & \sum_{s=0}^{k} \ln ^{s} \xi \sum_{\sigma=[(n-p) / 2]}^{\infty}\left(\xi^{n-2 \sigma} P_{k, s, \sigma, 0}(\eta ; \sigma)\right. \\
& \left.+\xi^{n-2 \sigma-1} P_{k, s, \sigma, 1}(\eta ; \sigma)\right) .
\end{aligned}
$$

Теорема 2.11. Ряды (1.22), построенные по функииям из теоремы 2.9, являются ф.а.р. задачи (1.21) при фиксированном разложсении $\bar{\lambda}_{\varepsilon}$ вида (1.8).

ДокАЗАТЕЛЬСТво стандартно [1].

Найдем теперь вид решений второй внутренней задачи в окрестности точки $(0 ; 1)$.

Для функций $w_{\varepsilon}^{+}\left(\xi, \eta_{+}\right):=z_{\varepsilon}\left(\varepsilon^{2} \xi, 1-\varepsilon^{2} \eta_{+}\right), v_{\varepsilon}^{+}\left(\xi, \eta_{+}\right):=u_{\varepsilon}\left(\varepsilon^{2} \xi, 1-\varepsilon^{2} \eta_{+}\right)$ищем асимптотику в виде

$$
w^{+}=\sum_{k=0}^{\infty} \varepsilon^{k} \sum_{l=0}^{k-2} w_{k, l}^{+} \ln ^{l} \varepsilon, \quad v^{+}=\sum_{k=0}^{\infty} \varepsilon^{k} \sum_{l=0}^{k-2} v_{k, l}^{+} \ln ^{l} \varepsilon .
$$

Для коэффициентов этих рядов получим задачи, аналогичные (1.23):

$$
\begin{cases}L_{\xi, \eta_{+}}^{+} w_{k, l}^{+}=p_{k, l}^{+}\left(\xi, \eta_{+}\right), \quad L_{\xi, \eta_{+}}^{-} v_{k, l}^{+}=q_{k, l}^{+}\left(\xi, \eta_{+}\right), & \left(\xi, \eta_{+}\right) \in \omega ; \\ w_{k, l}^{+}\left(\xi, \eta_{+}\right)=v_{k, l}^{+}\left(\xi, \eta_{+}\right)=0, & \left(\xi, \eta_{+}\right) \in \partial \omega .\end{cases}
$$

Из вида систем (1.23) и (2.11) следует, что поведение $w_{k, l}^{+}$аналогично поведению $v_{k, l}^{-}$, а $v_{k, l}^{+}$аналогичны $w_{k, l}^{-}$. Таким образом, $w_{k, l}^{+}$и $v_{k, l}^{+}$тоже описываются с помощью функций вида $\Xi^{(+, *)}(\cdot ;(n, p), k)$. При этом имеется небольшое отличие, связанное с видом правых частей, - теперь разложения функции $f$ сразу попадают в правую часть уравнения с оператором $L^{-}$, а не $L^{+}$, как в (1.23). Это приводит к незначительному изменению вида $w_{k, l}^{+}$и $v_{k, l}^{+}$, в частности, $0=w_{0,0}^{+}=v_{0,0}^{+}=v_{1,0}^{+}=v_{2,0}^{+}$. 
ТЕОРема 2.12. У системы (2.11) при фиксированном наборе $\left\{\lambda_{k, l}\right\}$ существуют решения $\left\{w_{k, l}^{+}\right\}$u $\left\{v_{k, l}^{+}\right\}$со следующей асимптотикой при $\xi+\eta_{+} \rightarrow+\infty$.

1) Eсли $k=4 m+2 n+\delta, m \in \mathbb{N}_{0}, n, \delta \in\{0,1\}$, mo

$$
w_{k, 0}^{+}=\stackrel{\circ}{\Xi}(k, 0,1,0,2,1), \quad v_{k, 0}^{+}=\stackrel{\circ}{\Xi}(k, 2,4,2,5,3) .
$$

2) Функции $w_{k, l}^{+}$имеют вид, аналогичный функциям $w_{k-2-l, 0}^{+}, a v_{k, l}^{+}$аналогичньь $v_{k-2-l, 0}^{+}$.

При этом функции $w_{2 k+1+\delta, 0}^{-}$u $v_{2 k+1+\delta, 0}^{-}$определены с точностью до слагаемых вида $R(\cdot ;(2 k+1,2 k+1))+e^{-\eta+S}(\cdot ;(2 k+1,2 k+1))$, однозначно определяемых многочленом степени $k$ от переменной $\eta_{+}$.

Справедлив и аналог теоремы 2.11.

Теорема 2.13. Ряды (2.10), построенные по функииям из теоремы 2.12, являются ф.а.р. задачи (1.1) в окрестности точки $(0 ; 1)$ при фиксированном разложении $\bar{\lambda}_{\varepsilon}$ вида (1.8).

\section{§ 3. Равномерная в $\Omega$ асимптотика функций $z_{\varepsilon}, u_{\varepsilon}$ и $\lambda_{\varepsilon}$}

Асимптотика функций $w_{k, l}^{\mp}, v_{k, l}^{\mp}$ и условия согласования позволяют окончательно определить функции $Z_{k, l}^{(\mp)}, U_{k, l}^{(\mp)}$, дающие разложение решений первой внутренней задачи.

В силу теорем 2.9 и 2.10 функции $w_{k, l}^{\mp}$ и $v_{k, l}^{\mp}$ можно разбить на два слагаемых

$$
w_{k, l}^{\mp}=\stackrel{1}{w_{k, l}^{\mp}}+\stackrel{2}{w_{k, l}^{\mp}}, \quad v_{k, l}^{\mp}=\stackrel{1}{v}_{k, l}^{\mp}+\stackrel{2}{v}_{k, l}^{\mp} .
$$

При этом ряды $Z$ и $U$ должны быть согласованы с рядами $\stackrel{1}{w}{ }^{\mp}$ и ${ }^{\mp}$, а $Z^{\mp}$ и $U^{\mp}-$ с $\stackrel{2}{w}^{\mp}$ и $\stackrel{2}{v}$ Ғ. Здесь и в дальнейшем вторая переменная у функций $w_{k, l}^{-}, v_{k, l}^{-}$будет обозначаться $\eta_{-}$вместо $\eta$.

Как и в [1], условия согласования имеют вид

$$
\begin{gathered}
\mathscr{A}_{N, \zeta, y}^{\mp} \mathscr{A}_{N, \xi, \eta_{\mp}} \stackrel{1}{w}^{\mp}=\mathscr{A}_{N, \xi, \eta_{\mp}} \mathscr{A}_{N, \zeta, y}^{\mp} Z, \\
\mathscr{A}_{N, \zeta, y}^{\mp} \mathscr{A}_{N, \xi, \eta_{\mp}}{ }^{\mp}=\mathscr{A}_{N, \xi, \eta_{\mp}} \mathscr{A}_{N, \zeta, y}^{\mp} U, \\
\mathscr{A}_{N, \zeta} \mathscr{A}_{N, \xi} \stackrel{2}{w}^{\mp}=\mathscr{A}_{N, \xi} \mathscr{A}_{N, \zeta} Z^{\mp}, \quad \mathscr{A}_{N, \zeta} \mathscr{A}_{N, \xi} \stackrel{2}{v}^{\mp}=\mathscr{A}_{N, \xi} \mathscr{A}_{N, \zeta} U^{\mp} .
\end{gathered}
$$

Здесь $\mathscr{A}_{N, \xi, \eta_{\mp}}$ - оператор взятия частичной суммы соответствующего асимптотического ряда в переменных $\left(\xi, \eta_{\mp}\right)$ при $\xi+\eta_{\mp} \rightarrow+\infty$, а $\mathscr{A}_{N, \zeta, y}^{-}$и $\mathscr{A}_{N, \zeta, y}^{+}$аналогичны $\mathscr{A}_{N, \xi, \eta_{\mp}}$, но при $(\zeta, y) \rightarrow(0 ; 0)$ и $(\zeta, y) \rightarrow(0 ; 1)$ соответственно. Операторы $\mathscr{A}_{N, \zeta}, \mathscr{A}_{N, \xi}$ отличаются от операторов $\mathscr{A}_{N, \zeta, y}^{\mp}, \mathscr{A}_{N, \xi, \eta_{\mp}}$ тем, что в разложениях переменными являются $\zeta$ и $\xi$, а $\eta_{\mp}-$ параметр. При этом равенства (3.1) справедливы, когда переменные связаны соотношениями $\varepsilon \xi=\zeta, \varepsilon^{2} \eta_{-}=y, \varepsilon^{2} \eta_{+}=1-y$.

Для наглядности воспользуемся таблицами согласования. Отметим, что при замене $\varepsilon \xi=\zeta, \varepsilon^{2} \eta_{-}=y, \varepsilon^{2} \eta_{+}=1-y$ переменная $\theta=\xi /\left(2 \sqrt{\eta_{\mp}}\right)$ сохраняет "свое" значение

$$
\theta^{-}=\frac{\zeta}{2 \sqrt{y}}, \quad \theta^{+}=\frac{\xi}{2 \sqrt{1-y}} .
$$


Фрагменты таких таблиц согласования рядов $U$ и $\stackrel{1}{v}-$ и рядов $Z$ и $\stackrel{1}{w}-$ приведены далее в таблицах 1 и 2 , так как остальные таблицы устроены аналогично (в таблицах для сокращения вместо $P(\cdot ;(k, n))$ записано $(k, n)$, а вместо $\mathscr{Q}(\theta ; n, m)$ $-[n, m])$.

Условие согласования дает вид особенностей функций $\stackrel{2}{Z_{k, l}^{(\mp)}}$ и $\stackrel{2}{U_{k, l}^{(\mp)}}$ в окрестности точек $(0 ; 0)$ и $(0 ; 1)$.

В окрестности точки $(0 ; 0)$ эти особенности имеют следующий вид:

$$
\left\{\begin{array}{l}
\stackrel{2}{Z}_{0,0} \sim \stackrel{2}{Z}_{1,0}=P(\cdot ;(2,0))+\cdots, \quad \stackrel{2}{Z}_{2,0}=P(\cdot ;(0, o))+\cdots ; \\
\stackrel{2}{Z}_{k, 0}=y^{1-k / 2}\left(\sum_{s=0}^{3} y^{s / 2} \Phi_{s}(\theta)+\mathscr{Y}(y, \theta, n(k))\right), \quad k \geqslant 3 ; \\
\stackrel{2}{Z}_{k, 1}=y^{(5-k) / 2} \Phi(\theta)+y^{3-k / 2} \mathscr{Y}(y, \theta, n(k)-1), \quad k \geqslant 3 ; \\
\stackrel{2}{Z}_{k, l} \sim \stackrel{2}{Z}_{k-l-1,1},
\end{array}\right.
$$

где

$$
\begin{aligned}
\mathscr{Y}(y, \theta, n):= & \left(\sum_{j=0}^{n(k)-1} \sum_{s=0}^{3} y^{2 j-2+s / 2} \sum_{\sigma=0}^{j} \mathscr{Q}(\theta ; 4 j-4+s, j-\sigma) \ln ^{\sigma} y\right. \\
& \left.+\sum_{s=0}^{\infty} y^{2 n(k)+s / 2} \sum_{\sigma=0}^{n(k)} \mathscr{Q}(\theta ; 4 n(k)+s, n(k)-\sigma) \ln ^{\sigma} y\right),
\end{aligned}
$$

a $n(k)=[(k-1) / 2]$

$$
\left\{\begin{array}{l}
\stackrel{2}{U}_{0,0} \sim \stackrel{2}{U}_{1,0}=P(\cdot ;(1, o))+\cdots, \stackrel{2}{U_{2,0}}=P(\cdot ;(0, o))+\cdots ; \\
\stackrel{2}{U}_{k, 0}=y^{2-k / 2} \mathscr{Y}(y, \theta, n(k)), \quad k \geqslant 3 ; \\
\stackrel{2}{U}_{k, 1}=y^{2-k / 2} \mathscr{Y}(y, \theta, n(k)-1), \quad k \geqslant 3 ; \\
\stackrel{2}{U}_{k, l} \sim \stackrel{2}{U}_{k-l-1,1} .
\end{array}\right.
$$

В окрестности точки $(0 ; 1)$ особенности $Z$ и $U$ меют следующий вид:

$$
\begin{aligned}
& \left\{\begin{array}{l}
\stackrel{2}{Z}_{0,0}=P(\cdot ;(1, o))+\cdots, \quad \stackrel{2}{Z}_{1,0} \sim \stackrel{2}{Z}_{2,0}=P(\cdot ;(0, o))+\cdots ; \\
\stackrel{2}{Z}_{k, 0}=y^{(5-k) / 2} \mathscr{Y}(y, \theta, n(k)), \quad k \geqslant 3 ; \\
\stackrel{2}{Z}_{k, 1}=y^{3-k / 2} \mathscr{Y}(y, \theta, n(k)-1), \quad k \geqslant 3 ; \\
2_{Z, l} \sim \stackrel{2}{Z}_{k-l-1,1},
\end{array}\right. \\
& \left\{\begin{array}{l}
\stackrel{2}{U_{0,0}}=P(\cdot ;(3,1))+\cdots, \stackrel{2}{U_{1,0}}=P(\cdot ;(2,0))+\cdots \\
\stackrel{2}{U_{2,0}}=P(\cdot ;(0, o))+\cdots \\
\stackrel{2}{U_{k, 0}}=y^{(3-k) / 2}\left(\sum_{s=0}^{3} y^{s / 2} \Phi_{s}(\theta)+\mathscr{Y}(y, \theta, n(k))\right), \quad k \geqslant 3 \\
\stackrel{2}{U}_{k, 1}=y^{(3-k) / 2} \Phi(\theta)+y^{3-k / 2} \mathscr{Y}(y, \theta, n(k)-1), \quad k \geqslant 3 \\
\stackrel{2}{U}_{k, l} \sim \stackrel{2}{U}_{k-l-1,1}
\end{array}\right.
\end{aligned}
$$




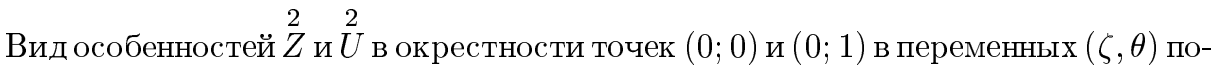
лучается из (3.2)-(3.5) путем следующих замен: $\Phi(\theta)-$ на $0, y^{r / 2}-$ на $\zeta^{r}, \mathscr{Q}_{r, k-j}-$ на $\mathscr{Q}_{0, k-j-1}$.

Стандартно показывается, что формальные ряды, получающиеся из рядов $w^{\mp}$ и $v^{\mp}$ после замены переменных и переразложения (строки рассмотренных таблиц согласования), являются ф.а.р. задачи (1.14).

ТЕОрема 3.1. Для фиксированного набора $\left\{\lambda_{k, l}\right\}$ существуют единственные решения задач (1.9)-(1.12), (1.16), (1.17), (1.23) и (2.11), вида (1.13), (3.2)(3.5) и описанного в теоремах 2.9 и 2.12, согласованные друг с другом.

ДокАЗАТЕЛЬство. Зафиксируем каким-либо образом многочлены, которые можно добавлять к $w_{k, l}^{\mp}$ и $v_{k, l}^{\mp}$ (например, нулевые). В силу теорем 2.9 и 2.12 однозначно найдутся все $w_{k, l}^{\mp}$ и $v_{k, l}^{\mp}$. При этом получим, что $\stackrel{2}{Z}_{0,0}^{-} \equiv 0 \equiv \stackrel{2}{=}{ }_{0,0}^{+}$. Это, в свою очередь, определяет начальные условия для $\stackrel{2}{Z}_{0,0}(\xi, 0) \equiv 0 \equiv \stackrel{2}{=}{ }_{0,0}(\xi, 1)$. Тогда по теореме 2.1 однозначно найдутся $\stackrel{2}{Z}_{0,0}$ и $\stackrel{2}{U_{0,0}}$, а по ним - и $\stackrel{2}{Z}_{0,0}^{+}$и $\stackrel{2}{U}_{0,0}^{-}$. Поскольку все условия согласованы, то эти функции не изменят $w_{0,0}^{\mp} \equiv 0 \equiv v_{0,0}^{\mp}$.

Затем определяются $\stackrel{2}{Z} \underset{1,0}{-} \equiv 0 \equiv \stackrel{2}{=} \stackrel{2}{+}_{1,0}^{+}$. По ним $\stackrel{2}{Z}_{1,0}, \stackrel{2}{U}_{1,0}$ и $\stackrel{2}{Z}_{1,0}^{+}, \stackrel{2}{U}_{1,0}^{-}$. Последние функции определяют “ядерные" добавки к $w_{1,0}^{\mp}$ и $v_{1,0}^{\mp}$, что приведет к фиксации двух первых столбцов и строк таблищ согласования и к изменению остальных клеток таблиц.

Далее процесс построения решений протекает аналогично: сначала определяются $\stackrel{2}{Z}_{k, l}^{-}$и $\stackrel{2}{U}_{k, l}^{+}$по уже определенной асимптотике при $\zeta \rightarrow 0$ и из условия экспоненциального убывания при $\eta_{\mp}+\infty$. Это определяет асимптотику при $\zeta \rightarrow 0$ начальных условий для функций $\stackrel{2}{Z}_{k, l}(\zeta, 0)$ и $\stackrel{2}{U}_{k, l}(\zeta, 0)$, после чего однозначно находятся $\stackrel{2}{Z}_{k, l}$ и $\stackrel{2}{U}_{k, l}$ с заданной асимптотикой. Эти функции, в свою очередь, приводят к однозначному нахождению $\stackrel{2}{Z}_{k, l}^{+}$и $\stackrel{2}{U_{k, l}^{-}}$, что, наконец, определяет функции $w_{k, l}^{\mp}$ и $v_{k, l}^{\mp}$ и очередные "ядерные" добавки.

Отметим, что функции $U_{k, l}^{-}$имеют в точке $(0 ; 0)$ следующие особенности:

$$
\begin{aligned}
& U_{k, 0}^{-}=\sum_{s=1}^{[(k-1) / 2]} \zeta^{4-k+s} P_{s}\left(\eta_{-}\right) \ln ^{s} \zeta, \quad k \geqslant 5, \\
& U_{k, l}^{-}=\sum_{s=l}^{[(k-1) / 2]} \zeta^{4-k+s} P_{s, l}\left(\eta_{-}\right) \ln ^{s-l} \zeta, \quad k \geqslant 5, \quad l \geqslant 1
\end{aligned}
$$

(здесь $P_{s}, P_{s, l}$ - некоторые многочлены).

Особенности у функции $U_{k, l}^{+}$такие же, как у $U_{k-3, l}^{-}$при $k \geqslant 8$. При остальных $k$ указанные функции особенностей в точке $(0 ; 0)$ не имеют.

В окрестности границы $x=1$ строятся ряды $Z_{1}\left(\zeta_{+}, y\right), Z_{1}^{\mp}\left(\zeta_{+}, \eta_{\mp}\right), U_{1}\left(\zeta_{+}, y\right)$ и $U_{1}^{\mp}\left(\zeta_{+}, \eta_{\mp}\right)$, аналогичные рядам $Z^{(\mp)}$ и $U^{(\mp)}$, а в окрестности точек $(1 ; 0)$ и $(1 ; 1)-$ 
ряды $w_{1}^{\mp}\left(\xi_{+}, \eta_{\mp}\right)$ и $v_{1}^{\mp}\left(\xi_{+}, \eta_{\mp}\right)$, аналогичные рядам $w^{\mp}$ и $v^{\mp}$. Здесь $\zeta_{+}:=(1-x) / \varepsilon$, a $\xi_{+}:=(1-x) / \varepsilon^{2}$.

Определим $z_{N}, u_{N}$ и $\lambda_{N}$ формулами

$$
\begin{aligned}
& \lambda_{N}:=\sum_{k=0}^{N} \sum_{l=0}^{k-2} \lambda_{k, l} \ln ^{l} \varepsilon, \\
& z_{N}=\mathscr{A}_{N, x, y}{ }_{\text {out }}^{z}+\mathscr{A}_{N, x} z^{-}+\mathscr{A}_{N, x} z^{+}+\mathscr{A}_{N, \zeta, y}^{-} Z+\mathscr{A}_{N, \zeta} Z^{-} \\
& +\mathscr{A}_{N, \zeta} Z^{+}+\mathscr{A}_{N, \zeta_{+}, y} Z_{1}+\mathscr{A}_{N, \zeta_{+}} Z_{1}^{-}+\mathscr{A}_{N, \zeta_{+}} Z_{1}^{+} \\
& +\mathscr{A}_{N, \xi, \eta} w^{-}-\mathscr{A}_{N, \xi, \eta}\left(\mathscr{A}_{N, \zeta, y}^{-} Z\right)-\mathscr{A}_{N, \xi}\left(\mathscr{A}_{N, \zeta} Z^{-}\right) \\
& +\mathscr{A}_{N, \xi, \eta_{+}} w^{+}-\mathscr{A}_{N, \xi, \eta_{+}}\left(\mathscr{A}_{N, \zeta, y}^{+} Z\right)-\mathscr{A}_{N, \xi}\left(\mathscr{A}_{N, \zeta} Z^{+}\right) \\
& +\mathscr{A}_{N, \xi^{+}, \eta} w_{1}^{-}-\mathscr{A}_{N, \xi^{+}, \eta}\left(\mathscr{A}_{N, \zeta_{+}, y}^{-} Z_{1}\right)-\mathscr{A}_{N, \xi_{+}}\left(\mathscr{A}_{N, \zeta_{+}} Z_{1}^{-}\right) \\
& +\mathscr{A}_{N, \xi^{+}, \eta^{+}} w_{1}^{+}-\mathscr{A}_{N, \xi^{+}, \eta^{+}}\left(\mathscr{A}_{N, \zeta_{+}, y}^{+} Z_{1}\right)-\mathscr{A}_{N, \xi_{+}}\left(\mathscr{A}_{N, \zeta_{+}} Z_{1}^{+}\right) \text {, }
\end{aligned}
$$

\begin{tabular}{|c|c|c|c|c|c|c|}
\hline 1 & & & & & & \\
\hline \multirow[t]{2}{*}{$1-\mu^{2}$} & $\begin{array}{c}\Omega_{2,0}^{+} \\
w^{+} \\
v^{+}\end{array}$ & $\begin{array}{c}\Omega_{1,0}^{+} \\
Z^{+}, Z \\
U^{+}, U\end{array}$ & $\begin{array}{c}\Omega_{0}^{+} \\
z^{+}, u^{+} \\
z, u\end{array}$ & $\begin{array}{c}\Omega_{1,1}^{+} \\
Z_{1}^{+}, Z_{1} \\
U_{1}^{+}, U_{1}\end{array}$ & $\begin{array}{c}\Omega_{2,1}^{+} \\
w_{1}^{+} \\
v_{1}^{+}\end{array}$ & \\
\hline & \multicolumn{2}{|c|}{$\begin{array}{c}\Omega_{1,0} \\
Z \\
U\end{array}$} & $\begin{array}{c}\Omega_{0} \\
\text { out } \\
z \\
\text { out } \\
u\end{array}$ & \multicolumn{2}{|c|}{$\begin{array}{c}\Omega_{1,1} \\
Z_{1} \\
U_{1}\end{array}$} & \\
\hline$\mu^{2}$ & $\begin{array}{l}\Omega_{2,0}^{-} \\
w^{-} \\
v^{-}\end{array}$ & $\begin{array}{c}\Omega_{1,0}^{-} \\
Z^{+}, Z \\
U^{+}, U\end{array}$ & $\begin{array}{c}\Omega_{0}^{-} \\
z^{-}, u^{-} \\
z, u\end{array}$ & $\begin{array}{c}\Omega_{1,1}^{-} \\
Z_{1}^{-}, Z_{1} \\
U_{1}^{-}, U_{1}\end{array}$ & $\begin{array}{l}\Omega_{2,1}^{-} \\
w_{1}^{-} \\
v_{1}^{-}\end{array}$ & \\
\hline 0 & \multicolumn{2}{|c|}{$\mu^{2}$} & $\mu \quad 1-\mu$ & \multicolumn{2}{|c|}{$1-\mu^{1}$} & 1 \\
\hline
\end{tabular}

$u_{N}$ определяется аналогично $z_{N}$.

Составные разложения $z_{N}$ и $u_{N}$ есть равномерная в $\Omega$ асимптотика задачи $(1.1)$ при фиксированном разложении $\lambda_{\varepsilon}=\bar{\lambda}_{\varepsilon}$. Это доказывается (как и в [1]) путем рассмотрения областей, в которых соответствующие ряды дают равномерную асимптотику системы и (или) граничных условий из (1.1) (см. рис. 1).

Рис. 1

ТЕОРемА 3.2. При фиксированном наборе $\left\{\lambda_{k, l}\right\}$ существует постоянная $\gamma$ такая, что

$$
\begin{cases}\mathscr{L} z_{N}+u_{N}-f=O\left(\varepsilon^{\gamma N}\right), \quad \mathscr{L}^{*} u_{N}-\lambda_{N} z_{N}=O\left(\varepsilon^{\gamma N}\right), & (x, y) \in \Omega \\ z_{N}(x, y)=O\left(\varepsilon^{\gamma N}\right), \quad u_{N}(x, y)=O\left(\varepsilon^{\gamma N}\right), & (x, y) \in \partial \Omega\end{cases}
$$

при $\varepsilon \rightarrow 0$ в смысле метрики пространств $C^{2}(\Omega), C^{2}(\partial \Omega)$ соответственно. 


\begin{tabular}{|c|c|c|c|c|c|}
\hline$U^{\cdot} \cdot v^{-}$ & $\varepsilon v_{1,0}^{1-}$ & $\varepsilon^{2} v_{2,0}^{-}$ & $\varepsilon^{3} v_{3,0}^{1-}$ & $\varepsilon^{4} \hat{v}_{4,0}^{-}$ & $\varepsilon^{4} \ln \varepsilon v_{4,1}^{-}$ \\
\hline$U_{0,0}$ & $\begin{array}{c}(1, o) \\
---- \\
\varepsilon(1, o)\end{array}$ & ---- & $\begin{array}{c}(3, o) \\
---- \\
\varepsilon^{3}(3, o)\end{array}$ & $\begin{array}{c}(4, o)+y^{2} \Phi \\
---- \\
\varepsilon^{4}\left((4, o)+\eta_{-}^{2} \Phi\right)\end{array}$ & ---- \\
\hline$\varepsilon U_{1,0}$ & $\begin{array}{c}\varepsilon(0, o) \\
---- \\
\varepsilon(0, o)\end{array}$ & $\begin{array}{c}\varepsilon(1, o) \\
---- \\
\varepsilon^{2}(1, o)\end{array}$ & $\begin{array}{c}\varepsilon(2, o) \\
---- \\
\varepsilon^{3}(2, o)\end{array}$ & $\begin{array}{c}\varepsilon(3, o) \\
---- \\
\varepsilon^{4}(3, o)\end{array}$ & ---- \\
\hline$\varepsilon^{2} U_{2,0}$ & ---- & ---- & $\begin{array}{l}\varepsilon^{2}(1, o) \\
----- \\
\varepsilon^{3}(1, o)\end{array}$ & $\begin{array}{c}\varepsilon^{2}((2, o)+y \Phi) \\
---- \\
\varepsilon^{4}\left((2, o)+\eta_{-} \Phi\right)\end{array}$ & ---- \\
\hline$\varepsilon^{3} U_{3,0}$ & ---- & ---- & $\begin{array}{l}\varepsilon^{3}(0, o) \\
----- \\
\varepsilon^{3}(0, o)\end{array}$ & $\begin{array}{c}\varepsilon^{3} y^{1 / 2}([1,1]+[1,0] \ln y) \\
---- \\
\varepsilon^{4} \eta_{-}^{1 / 2}[1,1]\end{array}$ & ---- \\
\hline$\varepsilon^{3} \ln \varepsilon U_{3,1}$ & ---- & ---- & ---- & $\begin{array}{l}\varepsilon^{3} y^{1 / 2}[1,0] \ln \varepsilon \\
\varepsilon^{3} y^{-1 / 2}[1,0] \ln \varepsilon\end{array}$ & $\begin{array}{c}\varepsilon^{3}(1, o) \ln \varepsilon \\
---- \\
\varepsilon^{3}(1, o) \ln \varepsilon\end{array}$ \\
\hline & & & & & \\
\hline
\end{tabular}

ТАБЛИЦА 1

Построенные функции $z_{N}$ и $u_{N}$, вообще говоря, не удовлетворяют условию (1.6). Подставив $u_{N}$ в $(1.6)$, получим

$$
\int_{\Omega}\left(2 u_{0}(x, y) \bar{u}_{N}(x, y)+\bar{u}_{N}^{2}(x, y)\right) d x d y=O\left(\varepsilon^{\alpha}\right)
$$

где $\bar{u}_{N}:=u_{N}-u_{0}$.

Обозначим $\widetilde{u}_{N}:=2 u_{0} \bar{u}_{N}+\bar{u}_{N}^{2}$. Найдя асимптотику интеграла из $(3.8)$, получим соотношения для $\left\{\lambda_{k, l}\right\}$.

Асимптотика этого интеграла находится аналогично тому, как это сделано в [6] с использованием доказанной там леммы 4.4. Поэтому здесь опишем этот процесс кратко.

В дальнейшем слагаемые вида $\sum_{s=1}^{n} \beta_{s} \mu^{a_{s}} \varepsilon^{b_{s}} \ln ^{c_{s}} \mu \ln ^{d_{s}} \varepsilon, c_{s}, d_{s} \in \mathbb{N}_{0}, a_{s}^{2}+$ $b_{s}^{2} \neq 0$, будем обозначать через $F(\mu, \varepsilon)$ или $F(\mu), b_{s}=0=d_{s}$.

Разобъем область $\Omega$ на подобласти, изображенные на рис. 1 , где $\mu \in\left[\varepsilon^{p} ; \varepsilon^{q}\right]$, $1 / 2<q<p<1$.

Рассмотрим, для примера, нахождение асимптотики интеграла от $\widetilde{u}_{N}$ в областях $\Omega_{0}$ и $\Omega_{0}^{-}$.

В области $\Omega_{0}$ равномерньм асимптотическим приближением $\bar{u}_{N}$ служит

$$
\mathscr{A}_{N, x, y}{ }^{\text {out }}-u_{0}=\sum_{k=1}^{N} \varepsilon^{k} \sum_{l=0}^{k-2} u_{k, l} \ln ^{l} \varepsilon \text {. }
$$




\begin{tabular}{|c|c|c|c|c|c|c|}
\hline$Z \cdot \cdot \stackrel{1}{w}^{-}$ & $\varepsilon^{2}{ }_{w}^{-}-0$ & $\varepsilon^{3} w_{3,0}^{-}$ & $\varepsilon^{4} \stackrel{1}{w}_{4,0}^{-}$ & $\varepsilon^{5} w_{5,0}^{-}$ & $\varepsilon^{5} \ln { }_{w}^{1}-{ }_{5,1}^{-}$ & $\varepsilon^{6} \stackrel{1}{w}_{6,0}^{-}$ \\
\hline$Z_{0,0}$ & $\begin{array}{c}(2, o) \\
+y \Phi \\
---- \\
\varepsilon^{2}((2, o) \\
\left.+\eta_{-} \Phi\right)\end{array}$ & $\begin{array}{c}(3, o) \\
---- \\
\varepsilon^{3}(3, o)\end{array}$ & $\begin{array}{c}(4,2) \\
+y^{2} \Phi \\
---- \\
\varepsilon^{4}((4,2) \\
\left.+\eta_{-}^{2} \Phi\right)\end{array}$ & $\begin{array}{c}(5,3) \\
---- \\
\varepsilon^{5}(5,3)\end{array}$ & ---- & $\begin{array}{c}(6,4) \\
+y^{3} \Phi \\
---- \\
\varepsilon^{6}((6,4) \\
\left.+\eta_{-}^{3} \Phi\right)\end{array}$ \\
\hline$\varepsilon Z_{1,0}$ & ---- & $\begin{array}{l}\varepsilon(((2, o) \\
+y \Phi) \\
---- \\
\varepsilon^{3}((2, o) \\
\left.+\eta_{-} \Phi\right)\end{array}$ & ---- & $\begin{array}{l}\varepsilon((4,2) \\
\left.+y^{2} \Phi\right) \\
---- \\
\varepsilon^{5}((4,2) \\
\left.+\eta_{-}^{2} \Phi\right)\end{array}$ & ---- & $\begin{array}{c}\varepsilon(5,3) \\
---- \\
\varepsilon^{6}(5,3)\end{array}$ \\
\hline$\varepsilon^{2} Z_{2,0}$ & $\begin{array}{c}\varepsilon^{2}((0, o) \\
\quad+\Phi) \\
---- \\
\varepsilon^{3}((0, o) \\
\quad+\Phi)\end{array}$ & $\begin{array}{l}\varepsilon^{2}(1, o) \\
---- \\
\varepsilon^{3}(1, o)\end{array}$ & $\begin{array}{l}\varepsilon^{2}((2, o) \\
+y \Phi) \\
---- \\
\varepsilon^{4}((2, o) \\
+\eta-\Phi)\end{array}$ & $\begin{array}{l}\varepsilon^{2}(3, o) \\
---- \\
\varepsilon^{5}(3, o)\end{array}$ & ---- & $\begin{array}{c}\varepsilon^{2}((4, o) \\
\left.+y^{2} \Phi\right) \\
---- \\
\varepsilon^{6}((4, o) \\
\left.+\eta_{-}^{2} \Phi\right)\end{array}$ \\
\hline$\varepsilon^{3} Z_{3,0}$ & $\begin{array}{c}\varepsilon^{3} y^{-1 / 2} \Phi \\
\varepsilon^{2} \eta_{-}^{-1 / 2} \Phi\end{array}$ & $\begin{array}{l}\varepsilon^{3}((0, o) \\
\quad+\Phi) \\
--- \\
\varepsilon^{3}((0, o) \\
\quad+\Phi)\end{array}$ & $\begin{array}{l}\varepsilon^{3}((1, o) \\
\left.+y^{1 / 2} \Phi\right) \\
---- \\
\varepsilon^{4}((1, o) \\
\left.+\eta_{-}^{1 / 2} \Phi\right)\end{array}$ & $\begin{array}{l}\varepsilon^{3}((2, o) \\
+y \Phi) \\
---- \\
\varepsilon^{5}((2, o) \\
\left.+\eta_{-} \Phi\right)\end{array}$ & ---- & $\begin{array}{c}\varepsilon^{3} y^{3 / 2}([3,1] \\
+[3,0] \ln y) \\
---- \\
\varepsilon^{6} \eta_{-}^{3 / 2}[3,1]\end{array}$ \\
\hline$\varepsilon^{3} \ln \varepsilon Z_{3,1}$ & ---- & ---- & ---- & ---- & $\begin{array}{l}\varepsilon^{3}((2, o) \\
+y \Phi) \ln \varepsilon \\
----- \\
\varepsilon^{5}((2, o) \\
+y \Phi) \ln \varepsilon\end{array}$ & $\begin{array}{c}\varepsilon^{3} \ln \varepsilon \\
\times y^{3 / 2}[3,0] \\
---- \\
\varepsilon^{6} \eta_{-}^{3 / 2} \\
\times[3,0] \ln \eta_{-}\end{array}$ \\
\hline & $\vdots$ & 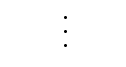 & $\vdots$ & & & \\
\hline
\end{tabular}

ТАБЛИЦА 2

Поскольку для функции $u \in C^{\infty}(\bar{\Omega})$ справедливо соотношение

$$
\int_{\Omega_{0}} u(x, y) d x d y=\int_{\mu}^{1-\mu} d y \int_{\mu}^{1-\mu} u(x, y) d x=\int_{\Omega} u(x, y) d x d y+F(\mu),
$$

Tо

$$
\int_{\Omega_{0}} \widetilde{u}_{N}(x, y) d x d y=\sum_{k=1}^{N} \varepsilon^{k} \sum_{l=0}^{k-2} \ln ^{l} \varepsilon\left(\int_{\Omega} u_{0}(x, y) u_{k, l}(x, y) d x d y+\widetilde{\gamma}_{k, l}\right)+o\left(\varepsilon^{\beta N}\right),
$$

где $\widetilde{\gamma}_{k, l}$ определяется членами разложения с меньшими номерами.

В силу (1.9) функции $z_{k, l}$ и $u_{k, l}$ можно представить в виде

$$
z_{k, l}=\lambda_{k, l} \bar{z}_{0}+\bar{z}_{k, l}, \quad u_{k, l}=\lambda_{k, l} \bar{u}_{0}+\bar{u}_{k, l}
$$


где $\bar{z}_{0}$ и $\bar{u}_{0}-$ решение задачи

$$
\mathscr{L}_{0} \bar{z}_{0}+\bar{u}_{0}=0, \quad \mathscr{L}_{0}^{*} \bar{u}_{0}-\lambda_{0} \bar{z}_{0}=z_{0}, \quad \bar{z}_{0}(x, 0)=0=\bar{u}_{0}(x, 1),
$$

а $\bar{z}_{k, l}$ и $\bar{u}_{k, l}$ определяются членами разложения с меньшими номерами.

Аналогично [6] можно показать, что при вьполнении условия (1.4)

$$
J_{0}:=\int_{\Omega} u_{0}(x, y) \bar{u}_{0}(x, y) d x d y>0
$$

Таким образом, вытекает

ЛЕмма 3.1. Справедливо следующее:

$$
\int_{\Omega_{0}} \widetilde{u}_{N}(x, y) d x d y=\sum_{k=1}^{N} \varepsilon^{k} \sum_{l=0}^{k-2} \ln ^{l} \varepsilon\left(\lambda_{k, l} J_{0}+\gamma_{k, l, 0}\right)+o\left(\varepsilon^{\beta_{0} N}\right),
$$

əде $\gamma_{k, l, 0}$ однозначно определяются членами разлоэсения с меньшими номерам⿻и.

В области $\Omega_{0}^{-}$равномерным асимптотическим приближением $\bar{u}_{N}$ служит

$$
\mathscr{A}_{N, x, y} \stackrel{\text { out }}{u}-u_{0}+\mathscr{A}_{N, x} u^{-}=\sum_{k=1}^{N} \varepsilon^{k} \sum_{l=0}^{k-2}\left(u_{k, l}+u_{k, l}^{-}\right) \ln ^{l} \varepsilon .
$$

Тогда $\widetilde{u}_{N}$ - сумма слагаемых вида $\varepsilon^{r} \ln ^{s} \varepsilon u(x, y), \varepsilon^{r} \ln ^{s} \varepsilon e^{-y / \varepsilon^{2}} u(x, y) u^{-}\left(x, y / \varepsilon^{2}\right)$ или $\varepsilon^{r} \ln ^{s} \varepsilon e^{-2 y / \varepsilon^{2}} u^{-}\left(x, y / \varepsilon^{2}\right)$, где $u(x, y) \in C^{\infty}(\bar{\Omega})$, a $u^{-}\left(x, \eta_{-}\right)$- многочлен по $\eta_{-}$ с коэффициентами, гладко зависящими от $x$. Но

$$
\begin{aligned}
& \int_{\Omega_{0}^{-}} u(x, y) d x d y=\int_{\mu}^{1-\mu} d y \int_{\mu}^{1-\mu} u(x, y) d x=F(\mu) \\
& \int_{\Omega_{0}^{-}} u(x, y) e^{-y / \varepsilon^{2}} u^{-}\left(x, \frac{y}{\varepsilon^{2}}\right) d x d y \\
& \quad=\varepsilon^{2} \int_{\mu}^{1-\mu} d x \int_{\mu}^{(\mu / \varepsilon)^{2}} u\left(x, \varepsilon^{2} \eta_{-}\right) e^{-\eta_{-}} u^{-}\left(x, \eta_{-}\right) d \eta_{-} \\
& =\varepsilon^{2} \int_{0}^{1} d x \int_{0}^{+\infty} u(x, 0) e^{-\eta_{-}} u^{-}\left(x, \eta_{-}\right) d \eta_{-}+F(\mu, \varepsilon), \\
& \int_{\Omega_{0}^{-}} e^{-2 y / \varepsilon^{2}} u^{-}\left(x, \frac{y}{\varepsilon^{2}}\right) d x d y=\varepsilon^{2} \int_{\mu}^{1-\mu} d x \int_{\mu}^{(\mu / \varepsilon)^{2}} e^{-2 \eta_{-}} u^{-}\left(x, \eta_{-}\right) d \eta_{-} \\
& =\varepsilon^{2} \int_{0}^{1} d x \int_{0}^{+\infty} e^{-2 \eta_{-}} u^{-}\left(x, \eta_{-}\right) d \eta_{-}+F(\mu, \varepsilon) .
\end{aligned}
$$

Таким образом, вытекает 
Лемма 3.2. Справедливо следующее:

$$
\int_{\Omega_{0}^{-}} \widetilde{u}_{N}(x, y) d x d y=\sum_{k=1}^{N} \varepsilon^{k} \sum_{l=0}^{k-2} \ln ^{l} \varepsilon \gamma_{k, l, 1}+o\left(\varepsilon^{\beta_{1} N}\right),
$$

где $\gamma_{k, l, 1}$ однозначно определяются членами разложсения с меньшими номерами (в частности: $\left.\gamma_{1,0,1}=0=\gamma_{2,0,1}\right)$.

В остальных областях нахождение асимптотики интегралов аналогично.

Таким образом, справедлива следуюшая теорема.

Teоpema 3.3. Cуществует такое $\beta>0$, что

$$
\int_{\Omega} \widetilde{u}_{N}(x, y) d x d y=\sum_{k=1}^{N} \varepsilon^{k} \sum_{l=0}^{k-2} \ln ^{l} \varepsilon\left(\lambda_{k, l} J_{0}+\gamma_{k, l}\right)+o\left(\varepsilon^{\beta N}\right),
$$

где $\gamma_{k, l}$ однозначно определяются членами всех асимптотических разлоэсений с меньшими номерами.

Тем самьм условие (3.8) с учетом теоремы 3.3 имеет вид

$$
\lambda_{k, l}=-\frac{\gamma_{k, l}}{J_{0}} .
$$

Теперь у нас есть все необходимое для построения равномерной в $\Omega$ асимптотики решения задачи (1.1), (1.2).

ТЕОРема 3.4. При выполнении условий (1.3), (1.4) существуют $\left\{\lambda_{k, l}\right\}$ такие, что $\lambda_{N}$ и составные асимптотические разложсения $z_{N}, u_{N}$ (см. (3.7)), построенные по решениям всех задач, определяющих функции этого разложения, являются равномерной в $\Omega$ асимптотикой набора $\left\{\lambda_{\varepsilon}, z_{\varepsilon}, y_{\varepsilon}\right\}$.

ДокАЗАТЕльство. Опишем процесс построения $\lambda_{k, l}$ и всех функций, входящих в составное асимптотическое разложение $z$ и $u$.

Положим $z_{0,0}:=z_{0}, u_{0,0}:=u_{0}, \lambda_{0,0}:=\lambda_{0}$ и $\lambda_{k, l}=0$ при всех $(k, l) \neq(0,0)$. Тогда по этим данным однозначно построятся все решения соответствующих задач, согласованные друг с другом.

По $u_{0}$ из (3.9) однозначно найдется $\lambda_{1,0}$. Теперь “подправим" решения всех этих задач с учетом нового $\lambda_{1,0}$.

В силу линейности всех систем при фиксированных $\lambda_{k, l}$ это приведет к добавлению "поправочных" слагаемых, начиная с внешнего разложения. При этом члены разложений с номерами $(0,0)$ не изменятся, а с номерами $(1,0)$ определятся однозначно с сохранением всех условий согласования.

После этого однозначно определится $\lambda_{2,0}$ и будут снова "подправлены" все члены разложения с не меньшими номерами и т.д.

Построенные таким образом $\lambda_{k, l}$ и все функции, входяшие в составное асимптотическое разложение, будут определять по формулам (3.7) и (3.6) функции $\lambda_{N}$, $z_{N}, u_{N}$, для которых в силу теорем $2.2,2.3$ и формулы (3.8) будут вьполняться все условия теоремы $1.1 \mathrm{c} \alpha=\gamma N$, где $\gamma$ - некоторая константа, определяемая лишь исходной задачей.

Автор выражает благодарность Е. Ф. Леликовой за внимание к работе и ценное обсуждение. 


\section{Список литературы}

1. Ильин A. М. Согласование асимтотических разложений решений краевых задач. М.: Наука, 1989.

2. Лионс Ж.-Л. Оптимальное управление системами, описьваемыми уравнениями с частными производньми. М.: Мир, 1972.

3. Данилин A.P. Асимптотика управлений для сингулярной эллиптической задачи // Докл. АН. 1999. Т. 369. №3. С. 305-308.

4. Данилин А.P. Аппроксимация сингулярно возмущенной эллиптической задачи оптимального управления // Матем. сб. 2000. Т. 191. № 10. С. 3-12.

5. Капустян B. E. Асимптотика ограниченных управлений в оптимальных эллиптических задачах // Докл. АН Украины. Сер. матем., естествозн., техн. науки. 1992. № 2. С. 70-74.

6. Данилин А.Р. Асимптотика ограниченных управлений для сингулярной эллиптической задачи в области с малой полостью // Матем. сб. 1998. Т. 189. № 11. С. 27-60.

7. Ильин A.M., Леликова E.Ф. Метод сращивания асимптотических разложений для уравнений $\varepsilon \Delta u-a(x, y) u_{y}=f(x, y)$ в прямоугольнике // Матем. сб. 1982. Т. 119. №3. C. 307-324.

8. Леликова E. Ф. Метод сращивания асимптотических разложений для уравнений $\varepsilon \Delta u-$ $a u_{z}=f$ в параллелепипеде // Дифференц. уравнения. 1978. Т. 14. №9. С. 1638-1648.

9. Калякин Л. А. Асимптотика решения системы двух линейных уравнений МГД с сингулярньп возмущением. І. Стандартная задача в эллиптическом слое // Дифференц. уравнения. 1982. Т. 18. №10. С. 1724-1738.

10. Ильин A. М. Пограничный слой // Совр. проблемы матем. Фундамент. направления. Т. 34. М.: ВИНИТИ, 1988. С. 175-214.

11. Васильева А. Б., Дмитриев М. Г. Сингулярные возмущения в задачах оптимального управления // Итоги науки и техники. Матем. анализ. Т. 20. М.: ВИНИТИ, 1982. C. 3-77.

12. Kokotovic P. V. Application of singular perturbation techniques to control problems // SIAM Rev. 1984. V. 26. № 4. P. 501-550.

13. Lions J. L. Perturbations singulleres dans les problemes aux limites eten controle optimal. Berlin: Springer-Verlag, 1973. (Lecture Notes in Math. V. 323).

14. Lions J.L. Asymptotic methods in the optimal control of distributed systems // Automatica J. IFAC. 1978. V. 14. P. 199-211.

15. Тихонов A.Н., Самарский А. А. Уравнения математической физики. М.: Наука, 1966.

16. Лебедев Н. Н. Специальные функции и их приложения. М.- Л.: Физматгиз, 1963.

17. Данилин A.P. О разрешимости одной системы уравнений в частных производных // Комплексный анализ, дифференциальные уравнения и смежные вопросы. II. Дифференциальные уравнения. Труды международной конференции. Уфа: ИМ с ВЦ, 2000. С. 53-57.

18. Карлсроу Г. С. Теория теплопроводности. М.-Л.: ГИТТЛ, 1947.

Институт математики и механики УрО РАН,

Поступила в редакцию

г. Екатеринбург

22.02 .2001 и 20.06 .2002

E-mail : dar@imm.uran.ru 